sites untereinander häufig verlinken, sich also gegenseitig referenzieren. Auch deshalb verstärkte sich das Forschungsinteresse hingehend einer Analyse der Netzwerke (oder: Relationen) alternativer Nachrichtenmedien. Da öffentliche Kommunikation auch Publika impliziert, liegt es nahe, als empirischen Wirklichkeitsausschnitt dafür Social-Media-Plattformen heranzuziehen, die Aufschluss über die Zielgruppen der Nachrichtenmedien, im Sinne von Abonnent_innen oder Follower_innen, geben. Darüber hinaus erscheint aufgrund der theoretischen Annahme einer digitalen Transformation der Öffentlichkeit, die vor allem von der Etablierung von Social-Media-Plattformen geprägt ist, die Analyse von Plattformstrukturen vielversprechender als eine weitere Analyse der One-Way-Kommunikation über die Websites. Wie bereits erwähnt, fiel die Wahl für diese Studie auf die Plattform Twitter, die sich nicht nur theoretisch für die Analyse der Relationen zwischen Nachrichtenmedien und weiteren Akteur_innen eignet, da gerade auf dieser Plattform ein Austausch von journalistischen, politischen wie auch Akteur_innen aus der Zivilgesellschaft antizipiert wird, sondern auch aufgrund forschungspraktischer Gründe. Twitter ermöglicht aus rein technischer Sicht eine detaillierte Analyse der Kommunikation von alternativen Nachrichtenmedien, die - wie in Kapitel VII.I beschrieben - auch auf Twitter aktiv sind und deren Beiträge durch Twitter-Nutzer_innen noch weiter verbreitet werden können. Die folgenden Kapitel VII.3 und VII.4 widmen sich den Forschungsfragen nach den Netzwerken bzw. Relationen alternativer Nachrichtenmedien untereinander und weiteren Akteur_innen auf Twitter.

\title{
VII.3 FF3: Twitter-Netzwerke alternativer Nachrichtenmedien
}

Nachdem im vorangegangenen Kapitel eine Definition alternativer Nachrichtenmedien und eine typologische Unterscheidung basierend auf dem Selbstverständnis der analysierten Medien erfolgten, stellt sich nun eine weitere zentrale Frage der vorliegenden Arbeit:

FF3: Wie sind alternative Nachrichtenmedien untereinander wie auch mit professionellen Nachrichtenmedien und weiteren Akteur_innen des öffentlichen Lebens auf der Digitalplattform Twitter vernetzt?

Die Frage nach der Vernetzung alternativer Nachrichtenmedien ergibt sich aus mehreren bisherigen theoretischen wie auch empirischen Befunden. Der digitale Strukturwandel der Öffentlichkeit ermöglicht demnach nicht nur neuen Kommunikator_innen, Öffentlichkeit herzustellen, sondern gerade die Plattformlogiken (oder «Social Media Logics»), vgl. hierzu van Dijck \& Poell (2013] evozieren eine Vernetzung unterschiedlicher Akteur_innen, die durch ebendiese Relatio- 
nen untereinander ihre Deutungsmacht erhöhen können, z. B. durch das Teilen oder Retweeten besonders prominenter Beiträge auf Plattformen. Castells (2007) spricht nicht umsonst von einer Netzwerkgesellschaft, in der gerade Gegenöffentlichkeiten (oder: «counter-powers») in Netzwerken untereinander agieren. So ist es - aus normativ-theoretischer Sicht - gerade bei alternativen Nachrichtenmedien von Relevanz, empirisch zu analysieren, inwiefern diese auf Plattformen ihre Netzwerke aufbauen - untereinander und auch mit ihren Publika, da eben (wie im vorangegangenen Kapitel ersichtlich wurde) nicht alle alternativen Nachrichtenmedien per se professionell journalistische Standards einzuhalten versuchen, sondern auch im gegenteiligen Sinne die hegemoniale Öffentlichkeit aus Politik und Medien der Lügen bezichtigen. Auch konnte in den bisherigen empirischen Analysen im Rahmen dieser Arbeit festgestellt werden, dass - zumindest auf den Websites - häufig auf weitere Nachrichtenmedien desselben Typs verlinkt wird, wodurch den Rezipierenden weitere Quellen offenbart werden, die die eigenen Ansichten bestärken.

Wie die ersten beiden Forschungsfragen ist auch die in diesem Kapitel fokussierte Frage explorativen Charakters. Somit wurden keine konkreten Hypothesen aufgestellt und nur grobe Annahmen oder Thesen formuliert, die sich aus bisherigen Analysen ergeben. Die weitere Verwendung des Begriffes «Netzwerk» bzw. «Netzwerke» bezieht sich auf zwei unterschiedliche Arten von Twitter-Netzwerken, einerseits Follower-Netzwerke, in denen untersucht wird, wie identifizierte Twitter-User_innen untereinander vernetzt sind, und andererseits Retweet-Netzwerke, um herauszufinden, inwiefern Twitter-Akteur_innen in Form geteilter Beiträge aufeinander Bezug nehmen. Bezugnehmend auf die Fragmentierungsthese (vgl. Kapitel II.3.3) wird erwartet, dass sich sowohl hinsichtlich der Follower- als auch der Retweetstrukturen homogene Communities bilden, die insbesondere von Communities der hegemonialen Öffentlichkeit (also z. B. «Mainstreammedien») abgetrennt sind:

These I (FF3): Alternative Nachrichtenmedien sind untereinander stark vernetzt. Dabei bilden sich vorwiegend Communities von alternativen Nachrichtenmedien eines Typs.

Infolgedessen wird davon ausgegangen, dass sich eine Zuordnung von Communities - also homogenen Clustern innerhalb der analysierten Netzwerke - daraus ergibt, welchem Alternativmedientyp diese zuzuordnen sind. Unter diesen «Typen» sind jene zu erwarten, die im Rahmen der zweiten Forschungsfrage (also des vorangegangenen Kapitels VII.2) identifiziert wurden. Dabei scheint es vor allem einen besonders präsenten Typ I zu geben, dessen Community als besonders gross erwartet wird: 
These 2 (FF3): Die Community des Typs «Aufdecker der Mainstreamlügen» ist besonders stark in den Netzwerken vertreten.

Im Rahmen der qualitativen Analyse zeichnete sich teilweise bereits ab, dass die unterschiedlichen Typen verschiedene politische Präferenzen haben. So richtet sich Typ I («Aufdecker der Mainstreamlügen») auffallend gegen linksorientierte Politik, was sich vor allem in der Ablehnung der Pro-Flüchtlingsdebatte äussert. Typ II («Verschwörung und Spiritualität») weist zumindest thematische Ähnlichkeiten mit Typ I auf, weshalb dieser in unmittelbarer Nähe innerhalb des Netzwerks erwartet wird. Typ III («Aufstand der Zivilgesellschaft») ist politisch links einzuordnen, da die sozialdemokratische oder sozialistische Linie hier per Selbstverständnis stark zum Ausdruck gebracht und rechtspopulistische Politik dezidiert abgelehnt wird. Letzteres zeigte sich auch bei Typ IV ( Die seriöse Alternative»). Daraus wird folgende These abgeleitet:

These 3 (FF3): Unterschiedliche Typen alternativer Nachrichtenmedien sind mit politischen Akteur_innen vernetzt, die ihrer Gesinnung entsprechen.

Weiter konnte im Rahmen der typologischen Unterscheidung festgestellt werden, dass sich die vier Alternativmedientypen in verschiedener Weise zum «Mainstream", also zu medialen und politischen Eliten, positionieren. Daraufhin ist die Annahme zu treffen, dass sich alternative Nachrichtenmedien je nach Grad der Ablehnung dieser hegemonialen Öffentlichkeit aus Politik und Medien in unterschiedlicher Weise mit diesen vernetzen bzw. von diesen distanzieren. Es wird erwartet, dass sich vor allem Typ I und II stark von Leitmedien und politischen Akteur_innen der Regierungen distanzieren, während sich Typ III und Typ IV vor allem von rechtsorientierter Politik distanzieren. Typ IV, der sich unter anderem als Ergänzung zum klassischen Journalismus betrachtet, könnte daher auch enge Verbindungen zum medialen Mainstream aufzeigen. Daraus ergibt sich folgende, offene These:

These 4 (FF3): Die vier Typen alternativer Nachrichtenmedien sind nicht in gleicher Weise von Communities medialen und politischen «Mainstreams» distanziert.

Abgesehen von diesen offen formulierten Thesen und deren Analyse besteht das Ziel dieses empirischen Schrittes darin, herauszufinden, wie alternative Nachrichtenmedien untereinander sowie mit dem professionellen Journalismus, mit politischen Akteur_innen und weiteren Akteur_innen des öffentlichen Lebens auf Social-Media-Plattformen vernetzt sind. Es handelt sich dabei um eine erste, deskriptive Betrachtung der Netzwerkstrukturen dieser Nachrichtenmedien, bevor 
im Rahmen der vierten Forschungsfrage (vgl. Kapitel VII.4) die Narrative oder der Sinn zwischen diesen Relationen qualitativ untersucht werden.

Durchgeführt wurden die Netzwerkanalysen - wie bereits in Kapitel VI.3 erörtert - mithilfe der Stream-API von Twitter unter Verwendung der Programmiersprache $\mathrm{R}$ und der Benutzeroberfläche RStudio. Durch die Erhebung sämtlicher URLs von alternativen und professionellen Nachrichtenmedien in Deutschland, Österreich und der Deutschschweiz konnte gewährleistet werden, dass im Zeitraum vom 15. September bis zum 27. Oktober 2019 wesentliche Tweets oder Retweets, die für dieses Projekt relevant sind, erhoben werden konnten, zumal Verlinkungen auf Websites nicht nur eine übliche Nutzer_innenaktivität auf Social-Media-Plattformen darstellen, sondern vor allem im Kontext von Nachrichten(-nutzung) zielführend erscheinen. Schliesslich ist nicht nur von Interesse, welche Beiträge (alternative) Nachrichtenmedien selbst posten und welche eigenen URLs sie verlinken, sondern auch, inwiefern weitere Twitter-Akteur_innen deren Nachrichten-URLs weiter streuen. Basierend auf der Suche von Nachrichten-URLs als zu suchende «keywords» wurden zwei Datensätze erstellt, bestehend aus Tweets, die einschlägige URLs implementiert haben (z. B. auch durch Retweets oder Replies). Der erste Datensatz beschränkt sich auf jene Tweets, in die die URLs alternativer Nachrichtenseiten eingebettet wurden - unabhängig davon, von welchen Absender_innen diese stammen. Ausserdem wurden in diesen Datensatz auch Tweets miteinbezogen, in denen die Screennames der Twitter-Auftritte von alternativen Newssites enthalten sind, da es sich hierbei um den zentralen Untersuchungsgegenstand handelt und dadurch ein noch grösserer Datenpool zustande kam. Der zweite Datensatz beinhaltet Tweets mit eingebetteten URLs von alternativen wie auch etablierten Nachrichtenmedien aus den drei Untersuchungsländern. Die beiden Datensätze wurden getrennt voneinander netzwerkanalytisch analysiert. Erstens, um einen detaillierten Blick auf die Netzwerkstrukturen von alternativen Nachrichtenmedien $\mathrm{zu}$ generieren, und zweitens, um durch den Vergleich mit etablierten Leitmedien der drei Länder in Erfahrung zu bringen, in welchem Ausmass alternativen Nachrichtenmedien eine Rolle in der gesamten öffentlichen Twitter-Kommunikation zukommt. Nur durch diese Gesamtbetrachtung ist es beispielsweise möglich, $\mathrm{zu}$ identifizieren, ob alternative Nachrichtenmedien und deren Followers abgetrennte Communities in den Netzwerken bilden, was auf eine Fragmentierung der Twitter-Öffentlichkeit oder auch Echokammernbildung hinweisen würde (vgl. Kapitel II.3.3).

Insgesamt wurden vier Netzwerkanalysen durchgeführt und mithilfe des Visualisierungstools Gephi dargestellt. $\mathrm{Zu}$ betrachtende Masse im Kontext der Analysen sind auf Netzwerkebene die Dichte des Netzwerks, die durchschnittliche Pfadlänge und etwaige Cluster, also homogene Gruppen oder Communities, basierend auf dem Modularitätswert. Die Dichte des Netzwerks gibt basierend auf dem Kennwert der Kantendichte das Verhältnis von tatsächlich existierenden 
Verbindungen oder Beziehungen zwischen Akteur_innen und der maximalen Anzahl möglicher Verbindungen im Netzwerk an. Je höher der Wert, desto dichter ist das Netzwerk und umso stärker sind die einzelnen Knoten miteinander vernetzt ein Wert von 1 wäre somit erreicht, wenn alle Knoten miteinander verbunden sind. Die Pfadlänge gibt den durchschnittlich kürzesten Weg von einem Knoten zu jedem anderen Knoten des Netzwerks an. Je höher dieser Wert ist, desto grösser ist die Wahrscheinlichkeit, dass einzelne zentrale Knoten zwischen den restlichen Knoten vermitteln. Das Modularitätsmass gibt Aufschluss darüber, inwiefern das Netzwerk in unterschiedliche Teilgruppen (Cluster oder Communities) ausdifferenziert werden kann. Ein hoher Modularitätswert deutet darauf hin, dass dichte Verbindungen innerhalb einer Community und nur wenige Verbindungen gegenüber Knoten anderer Communities existieren. Auf der Ebene der einzelnen Knoten stellen die Zentralitätsmasse wesentliche Kennwerte für deren Einordnung dar. Der Eingangsgrad (indegree) kennzeichnet, wie viele Verbindungen sich bei einem Knoten treffen, also wie viele weitere Knoten auf ihn Bezug nehmen. Der Ausgangsgrad (outdegree) wiederum misst die ausgehenden Verbindungen eines Knotens zu anderen Knoten. Insgesamt misst der Grad (degree) die Summe an einund ausgehenden Verbindungen. Die Nähezentralität (closeness centrality) gibt die Distanz zu allen weiteren Knoten im Netzwerk an. Je höher der Wert ist (maximal 1), desto eingebundener ist der Knoten im Gesamtnetzwerk. Die Zwischenzentralität (betweenness centrality) zeigt an, inwiefern ein Knoten vermittelnd im Gesamtnetzwerk fungiert. Hohe Zwischenzentralitätswerte deuten darauf hin, dass der jeweilige Knoten zwischen unterschiedlichen Communities eine verbindende Rolle einnimmt. Eine hohe Eigenvektorzentralität (maximal 1) legt nahe, dass ein Knoten umso bedeutsamer im Gesamtnetzwerk ist, je näher seine Verbindungen $\mathrm{zu}$ weiteren relevanten Knoten (im Sinne hoher Grade) sind (Bauschmann \& Ahnert, 2016).

Für beide Datensätze wurden jeweils sowohl die Follower-Netzwerke als auch die Retweet-Netzwerke analysiert. Diese beiden Netzwerkstrukturen werden in den folgenden Unterkapiteln beschrieben und visuell dargestellt. Dabei werden jeweils die Netzwerke alternativer Nachrichtenmedien mit den Gesamtnetzwerken gegenübergestellt, die deutschsprachige professionelle Nachrichtenmedien inkludieren. Neben einer inhaltlichen Beschreibung der Netzwerke werden jeweils die Masse auf Netzwerkebene (Kantendichte, Pfadlänge, Modularität) angeführt; die Beschreibung einzelner Knoten erfolgt vor allem inhaltlich unter Anführung des Eingangsgrades, als Mass dafür, wie definitionsmächtig der Knoten im Netzwerk ist. Alle weiteren Zentralitätswerte werden in Anhang 2 angeführt. 


\section{VII.3.1 Follower-Netzwerkstrukturen}

Wie in Kapitel VI.3 erläutert, handelt es sich bei sogenannten Follower-Netzwerken um relationale Verbindungen von Twitter-Nutzer_innen und deren Abonnent_innen, die aufder Plattform Twitter als Followers bezeichnet werden. Jede_r Twitter-User_in kann entsprechend sein/ihr eigenes Netzwerk an Akteur_innen aufbauen, da diese Verbindungen aktiv und meist ohne Zustimmung des gefolgten Akteurs/der gefolgten Akteurin eingegangen werden. Von Interesse sind - aus einer netzwerkanalytischen Betrachtung - Ähnlichkeiten in Followerstrukturen. Darunter ist zu verstehen, dass bestimmte Akteur_innen in der Twitter-Öffentlichkeit über ähnliche bzw. sich überschneidende Follower_innen verfügen. Dies ist beispielweise dann der Fall, wenn Twitter-User_innen über ähnliche Merkmale verfügen, z. B. hinsichtlich der politischen Gesinnung oder bestimmter Interessen, und somit ein ähnliches Publikum ansprechen. Dies könnte potentiell zu einer Fragmentierung der (Twitter-)Öffentlichkeit führen, wenn Gruppen bzw. Communities mit ähnlichen Interessen resultieren und diese voneinander abgetrennt sind, was in der aktuellen Forschung häufig als «Echokammern» bezeichnet wird (vgl. Kapitel II.3.3). Aus normativer Sicht ist es problematisch, wenn sich ebendiese Communities in ihren eigenen Ansichten weiter bestärken und mit anderen Positionen nicht mehr in Berührung kommen. Häufig bleiben allerdings die Gründe unreflektiert, warum bestimmten Akteur_innen auf Plattformen gefolgt wird, diese also abonniert werden, zumal das reine Rezipieren von Inhalten gewisser Akteur_innen nicht unbedingt mit einer Zustimmung dieser einhergehen muss. Aus diesem Grund wird - insbesondere im Zuge der Bearbeitung von Forschungsfrage 4 (vgl. Kapitel VII.4) ein Fokus auf die qualitative Interpretation von relationalen Verbindungen gelegt.

Nachfolgend werden zunächst die Follower-Netzwerke deutschsprachiger alternativer Nachrichtenmedien wie auch alternativer und professioneller Nachrichtenmedien illustriert und beschrieben.

\section{VII.3.1.1 Follower-Netzwerk alternativer Nachrichtenmedien}

Das Follower-Netzwerk «alternative Nachrichtenmedien» bezieht sich auf jene Unique Users, die aus dem Datensatz extrahiert werden konnten, der sich auf die URLs und Screennames alternativer Nachrichtenmedien im gesamten DACHRaum beschränkt. Unter Unique Users werden in diesem Zusammenhang alle Twitter-User_innen verstanden, die einen Tweet im Untersuchungszeitraum abgesendet haben und somit URLs alternativer Nachrichtenmedien verlinkt haben, einschlägige Tweets retweetet haben, oder als alternatives Nachrichtenmedium (basierend auf der induktiv erhobenen Liste, vgl. Anhang 1a) markiert wurden (Mentions). Insgesamt wurden 49'032 Unique Users im Datensatz identifiziert, davon konnten für 45'195 User_innen deren Twitter-Follower_innen automati- 
siert erhoben werden ${ }^{133}$. Daraus resultierten insgesamt 4'058'296 Followers. Alle Accounts und deren Followers («Knoten» und «Kanten») wurden in einer tabellarischen Auflistung festgehalten, die unter Verwendung von R (Package «igraph») und dem Visualisierungsprogramm Gephi ausgewertet wurden. Für die folgende Abbildung 20 wurden schwache Knoten aus der Datengrundlage ausgeschlossen, die anhand eines geringen Degree-Wertes, also der Anzahl an Verbindungen zu weiteren Accounts, identifiziert wurden. Für die vorliegende Abbildung wurden Knotenpunkte mit einem Degree kleiner 400 ausgeschlossen, somit resultierten 5'191 Knoten und 1'599'528 Kanten, die zur graphischen Aufbereitung unter Verwendung des ForceAtlas-2-Algorithmus des Netzwerks dienten. Alle ausgewiesenen Kennwerte und Interpretationen beziehen sich auf sämtliche Daten des Netzwerks.

\section{Abbildung 20: Twitter-Follower-Netzwerk alternativer Nachrichtenmedien (Eigene Darstellung)}

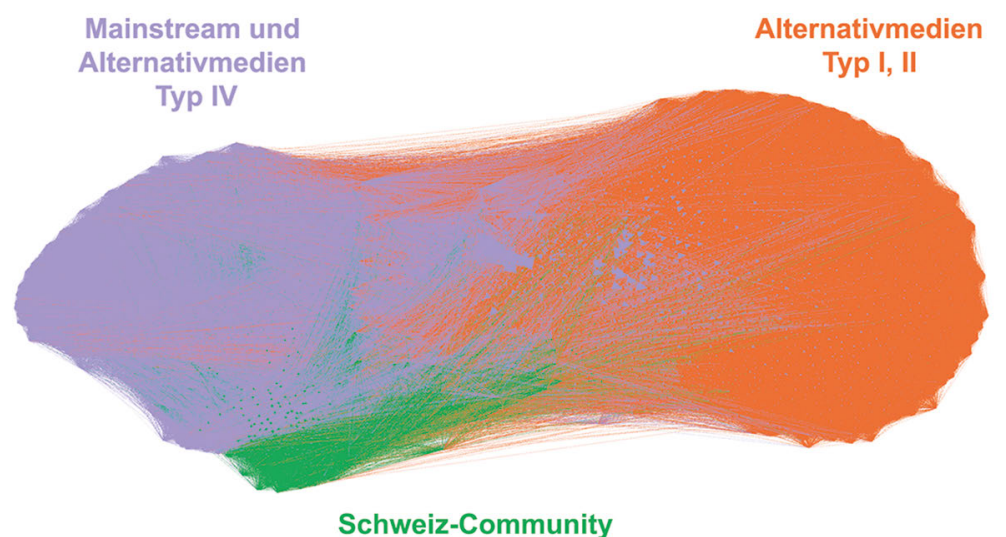

Das Netzwerk hat eine Kantendichte von 0,002 (wonach gesamthaft betrachtet alle Knoten des Netzwerks nur lose miteinander in Verbindung stehen), eine mittlere Pfadlänge (im Sinne von durchschnittlich verbindenden Knoten zwischen zwei Knoten) von 3,261 und einen Modularitätswert von 0,332. Insgesamt konnten

133 Dass nicht für alle Unique Users die Followerbeziehungen erhoben werden konnten, kann unterschiedliche Gründe haben. So können beispielsweise für User_innen, die ihren Account auf Twitter zwischen dem Erhebungszeitraum der Tweets und dem Tracking der Followers gelöscht haben, im Nachgang keine Daten erhoben werden. Auch wurden bereits während der Erhebung der Followers Accounts übersprungen, die geschützt sind, über keine Followers verfügen, oder eine Follower-Anzahl über 75'000 aufweisen. Hier könnte es sich ggf. um Social Bots handeln. 
mittels Louvain-Algorithmus (Blondel, Guillaume, Lambiotte \& Lefebvre, 2008) 28 Communities identifiziert werden, von denen drei die grössten Anteile im Netzwerk einnehmen. Die fokussierten Communities wurden jeweils farblich gekennzeichnet, wobei sich hinter der Farbgebung kein Code verbirgt; diese wurde zufällig gewählt. Eine Community bezieht sich dabei auf ähnliche Follower-Beziehungen innerhalb dieser und grenzt sich von den anderen Communities durch eine unterschiedliche Followerstruktur ab. Die Beschriftungen innerhalb der Visualisierung des Netzwerks beziehen sich auf die identifizierten Communities. Besonders starke Knoten innerhalb der einzelnen Communities und deren zentralen Kennwerte sind in Anhang $2 a$ aufgeführt. Dabei handelt es sich vorwiegend um alternative und professionelle Nachrichtenmedien der drei Untersuchungsländer sowie allenfalls weitere Akteur_innen des öffentlichen Lebens (z. B. Politiker_innen, soziale Bewegungen), die für eine einfachere Interpretation des Netzwerks dienen. Private Accounts von Twitter-User_innen verfügen erstens üblicherweise über nicht so hohe Reichweiten (im Sinne von der Anzahl Follower_innen oder Retweets) und werden zweitens aus datenethischen Gründen nicht explizit angeführt.

Auf den ersten Blick wird deutlich, dass zumindest aus struktureller Sicht von keiner starken Polarisierung zwischen den Communities - die sich allesamt um alternative Nachrichtenmedien als zentrale Knotenpunkte bilden - auszugehen ist, da das Netzwerk in sich geschlossen ist und die drei fokussierten Communities keine getrennten Cluster (im Sinne von «Echokammern», vgl. Kapitel II.3.3) bilden. Umso interessanter ist ein Blick auf den horizontalen Verlauf der Knotenpunkte innerhalb des Netzwerks, die eine politische Positionierung widerspiegeln. Die lilafarbene Community $(36,84 \%)$ repräsentiert demnach eine politische Links- oder Mitteorientierung, was sich deutlich an den Nachrichtenmedien und weiteren Twitter-Akteur_innen erkennen lässt, die als starke Knotenpunkte innerhalb des Clusters gekennzeichnet sind. Auffallend ist dabei, dass jene Akteur_innen, die weiter links im Netzwerk platziert sind, auch politisch weiter links einzuordnen sind. Bei dieser Einordnung hilft vor allem die typologische Untersuchung alternativer Nachrichtenmedien im Rahmen der Ausarbeitung von Forschungsfrage 2 (vgl. Kapitel VII.2), die es nun rein deskriptiv ermöglicht, das Selbstverständnis der untersuchten Alternativmedientypen in die Interpretation des Netzwerks zu integrieren. So liegt beispielsweise der Twitter-Account von Volksverpetzer (Eingangsgrad: 6'065) auf der linken Seite des Netzwerks, wie auch der Account Uebermedien (Eingangsgrad: $3^{\prime} 750$ ) oder Correctiv.org (Eingangsgrad: 4'661) und BILDblog (Eingangsgrad: 6'784). Alle drei Seiten haben gemein, dass sie sich als eine Art «Media-Watchdogs» präsentieren und nicht als Nachrichtenmedien in dem Sinne zu kategorisieren sind, dass sie in regelmässigen Abständen 
selbständig Nachrichten verbreiten. ${ }^{134}$ Die Medien setzen sich demnach das Ziel, einen Metadiskurs über Medien zu führen, verbunden mit einer Aufklärungsrolle oder Korrektivfunktion gegenüber desinformativer Berichterstattung, wie an diesem Beispiel von Volksverpetzer ersichtlich:

Uns ärgern Hass, Hetze, Fake News und Verschwörungstheorien in Social Media genau wie alle anderen. Deshalb wollen wir etwas dagegen tun. Als Anti-Fake-NewsBlog versuchen wir, die tolle Arbeit der vielen großartigen Faktenchecker*innen mit kreativen Aktionen, Witz, Satire und ebenso ausführlichen Recherchen zu ergänzen. ${ }^{135}$

Bezugnehmend auf die typologische Einordnung alternativer Nachrichtenmedien in Kapitel VII.2 würden die drei Beispiele am ehesten in den Typ IV «Die seriöse Alternative» fallen, sofern sie als Nachrichtenmedium klassifiziert werden $^{136}$. Politisch ebenfalls in das linke Spektrum einzuordnen, ist der Twitter-Account Fridays for Future (Eingangsgrad: 5'127), der die deutsche soziale Bewegung zum Klimaschutz repräsentiert und aus netzwerkanalytischer Sicht über ähnliche Followerstrukturen verfügt wie die eben genannten Alternativmedien. Es sind aber nicht nur die Accounts dieses Alternativmedientyps, die eine ähnliche Followerstruktur aufweisen, sondern auch professionelle Nachrichtenmedien und Journalist_innen, die Teil dieser Community sind und über ähnliche Follower_innen verfügen. So beispielsweise der Account des österreichischen Journalisten und ORF-Moderatoren Armin Wolf (Eingangsgrad: 4'040), der innerhalb des «Mainstreams» in dieser Community offenbar eher politisch linksorientierte Twitter-User_innen anspricht und für seine Kritik an rechtspopulistischer Politik bekannt ist ${ }^{137}$. Mittig im Netzwerk ersichtlich - und auf der rechten Seite der farblich in lila gekennzeichneten Community - finden sich professionelle Leitmedien aus den drei deutschsprachigen Untersuchungsländern, darunter beispielsweise die deutsche Tagesschau als einer der stärksten Knotenpunkte innerhalb der Community mit einem Eingangsgrad von 10'583 und somit den meisten eingehenden Verbindungen von anderen Twitter-Akteur_innen, gefolgt von der Süddeutschen Zeitung (Eingangsgrad: 8'903), Die Welt (Eingangsgrad: 6'515) oder ZDFheute (Eingangsgrad: 5'852). Ein weiterer starker Knoten in dieser Reihe ist

134 So wie es im Rahmen der typologischen Einordnung (Forschungsfrage 2; vgl. Kapitel VII.2) vorausgesetzt war.

135 https://www.volksverpetzer.de/ueber-uns/ (Stand: 22.07.2020)

136 Auch an dieser Stelle sei darauf hingewiesen, dass typologische Einordnungen auch Graubereiche implizieren. Das digitale Mediensystem ist im Zuge der digitalen Transformation so dispers geworden, dass eine trennscharfe Kategorisierung in Medientypen nicht immer möglich ist.

137 Z. B. https://www.arminwolf.at/2015/11/07/was-darf-ein-interviewer-im-orf/ (Stand: 22.07. 2020) 
der Twitter-Account von Der Postillon (Eingangsgrad: 11'157), der kein klassisches Nachrichtenmedium repräsentiert, sondern als deutsches Satire-Magazin auftritt. Die lilafarbene Community lässt sich demnach am besten unter den Titel «Mainstreammedien und Alternativmedien des Typs IV» fassen.

Wie in Abbildung 20 dargestellt, sind Verbindungen zwischen den drei visualisierten Communities ersichtlich, weshalb anzunehmen ist, dass sich die Followercommunities in gewissem Ausmass überschneiden. So zeigt sich beispielsweise, dass die NZZ (Eingangsgrad: 5'535) mit ihrer konservativen Haltung und der Twitter-Auftritt der Weltwoche (Eingangsgrad: 1'222) nahe Knotenpunkte innerhalb des Netzwerks darstellen, obgleich sie unterschiedlichen Communities angehören. Dies ist nicht verwunderlich, da die in grün markierte Community die Schweizer Nachrichtenmedien repräsentiert, zu der inhaltlich auch die NZZ zählen würde. Dennoch kann der grün gekennzeichnete Cluster (3,45 \%) klar als die «Schweiz-Community» bezeichnet werden, in der sich neben der Weltwoche auch die Republik (Eingangsgrad: 1'605) und der Tages-Anzeiger (Eingangsgrad: I'428) wiederfinden. Die ist vor allem deswegen interessant, da alle drei genannten Nachrichtenmedien kategorisch in unterschiedliche Nachrichtenmedientypen einzuordnen sind. So repräsentiert die Weltwoche ein alternatives Nachrichtenmedium, das sich klar gegen den Mainstream richtet und typologisch in Typ I («Aufdecker der Mainstreamlügen») einordnen liesse, während die Republik ein Beispiel für Typ IV darstellt («Die seriöse Alternative») und der Tages-Anzeiger ein klassisches Schweizer Leitmedium repräsentiert. Bezogen auf die Followerstrukturen innerhalb dieser Community ist daher anzunehmen, dass Schweizer Nachrichtenmedien - unabhängig von ihrer journalistischen Ausrichtung - über ähnliche Twitter-Follower_innen verfügen. Dies muss gleichzeitig jedoch nicht bedeuten, dass Follower der Republik und der Weltwoche beide Medien unterstützen, alleine aus dem Grund, dass sie womöglich beide Medien auf Twitter abonniert haben. Die NZZ hingegen, dem lilafarbenen Cluster zugeordnet, scheint ein internationaleres Publikum (auch aus Deutschland und Österreich) anzusprechen. Insbesondere im lilafarbenen Cluster sind Nachrichtenmedien und Akteur_innen aus allen drei deutschsprachigen Ländern vorzufinden (z. B. Journalist Armin Wolf aus Österreich, die Schweizer NZZ und die deutsche Tagesschau).

Die in orange gekennzeichnete Community (14,35\%) spiegelt dabei jene alternativen Nachrichtenmedien wider, die in Anlehnung an die typologische Einordnung (vgl. Kapitel VII.2) Typ I («Aufdecker der Mainstreamlügen») und Typ II («Verschwörung und Spiritualität») zuzuordnen sind. Gleichzeitig verdeutlichen die Netzwerkstrukturen, dass jene beiden Typen einen wesentlichen Teil der resonanzreichen (in Bezug auf ihre Follower_innen-Anzahl) Alternativmedien in der deutschsprachigen Twittersphäre ausmachen, während sich Typ IV («Die seriöse Alternative») eine Follower-Community mit den professionellen «Mainstream»-Medien teilt und alternative Nachrichtenmedien des Typs III («Aufstand 
der Zivilgesellschaft») keine starken Knotenpunkte innerhalb des Netzwerks aufweisen und auch über keine eigene Community verfügen. Ein besonders starker Knoten in diesem Cluster ist der Twitter-Account von Roland Tichy (mit einem Eingangsgrad von 6'082), der gleichzeitig Macher des alternativen Nachrichtenmediums Tichys Einblick ist (Eingangsgrad: 5'268), das Typ I («Aufdecker der Mainstreamlügen») zugeordnet werden kann. Neben weiteren Alternativmedien dieses Typs, die starke Knoten im Netzwerk repräsentieren (z. B. Achgut.com (Achse des Guten) (Eingangsgrad: 4'747), Junge Freiheit (Eingangsgrad: 4'470) und Hartes Geld (Eingangsgrad: 3'800), spiegeln das COMPACT Magazin (Eingangsgrad: 3'719) oder Russia Today Deutsch (Eingangsgrad: 3'787) eher verschwörungstheoretische Medien wider (Typ II). Abgesehen von Nachrichtenmedien innerhalb des orangefarbenen Clusters konnten ferner mehrere politische Accounts identifiziert werden, vorwiegend aus den Reihen der AfD (Eingangsgrad: 5'115) und ihren Politiker_innen, z. B. Beatrix von Storch (Eingangsgrad: 4'413) und Björn Höcke (Eingangsgrad:3'239), oder auch der Jurist Joachim Steinhöfel (Eingangsgrad: 5'197), der die AfD bereits vor Gericht vertrat ${ }^{138}$ und als Autor in Medien wie Tichys Einblick und Die Achse des Guten tätig ist. Ebenso finden sich in diesem Cluster die Accounts des Schweizer SVP-Politikers Roger Köppel (Eingangsgrad: 3'061) und von Akteur_innen der Identitären Bewegung ${ }^{139}$, wie z. B. Martin Sellner (Eingangsgrad: $3^{\prime} 099$ ).

Diese Netzwerkanalyse zeigte jene Followercommunities, die bestimmten Nachrichtenmedien im deutschsprachigen Raum folgen. Fokussiert wurden hierbei die Netzwerkbeziehungen jener Accounts, die anhand des Trackings alternativer Nachrichtenmedien auf Twitter identifiziert werden konnten. Um den Blick zu weiten, wurde ein weiteres Follower-Netzwerk erstellt, das explizit auch Accounts professioneller Nachrichtenmedien (dem «Mainstream») in der Analyse berücksichtigt hat. Dieses wird nachfolgend präsentiert und dem anderen gegenübergestellt.

\section{VII.3.1.2 Follower-Netzwerk alternativer und professioneller Nachrichtenmedien}

Bereits im eben beschriebenen Follower-Netzwerk wurde deutlich, dass Follower_ innen alternativer Nachrichtenmedien teilweise auch professionellen Nachrichtenmedien auf Twitter folgen. Um einen Überblick über die Followerstrukturen sämtlicher deutschsprachiger Nachrichtenmedien (alternativ wie auch professionell) zu

138 Z. B. https://www.zeit.de/politik/deutschland/2020-06/andreas-kalbitz-urteil-afd-mitglied schaft/seite-2 (Stand: 22.07.2020)

139 Twitter-Accounts von Anhänger_innen der Identitären Bewegung wurden beispielsweise im Juli 2020 aufgrund von Richtlinienverstössen und extremistischem Gedankengut gelöscht: z. B. https://www.tagesspiegel.de/politik/twitter-loescht-konten-von-50-nationalistenidentitaere-bewegung-in-deutschland-und-oesterreich-verliert-accounts/25997090.html (Stand 22.07.2020) 
erhalten, bezieht sich das folgende Netzwerk auf die identifizierten User_innen des kompletten Datensatzes, der sowohl alternative als auch professionelle Nachrichtenmedien als Suchworte inkludierte. Auch in diesem Fall beschränkt sich die Visualisierung des Netzwerks auf die stärksten Knotenpunkte; Knoten mit einem Degree unter 800 wurden hierfür ausgeschlossen, um eine Übersichtlichkeit zu gewährleisten. Sämtliche angeführten Kennwerte beziehen sich jedoch auf das Gesamtnetzwerk. Von den insgesamt 143'800 Knoten und 18'233'015 Kanten bezieht sich die folgende Abbildung 21 auf die stärksten 10'757 Knoten und ihre insgesamt 4'272'676 Kanten, also Beziehungen zu weiteren Twitter-Akteur_innen. Die Kantendichte beträgt 0,001 , die mittlere Pfadlänge 26,12 . Von den insgesamt 141 identifizierten Communities (Louvain-Algorithmus) mit einem Modularitätswert von $0,469^{140}$ stechen im visualisierten Netzwerk vor allem vier wiederum farblich markierte Communities hervor.

Abbildung 21: Twitter-Follower-Netzwerk alternativer und professioneller Nachrichtenmedien (Eigene Darstellung)

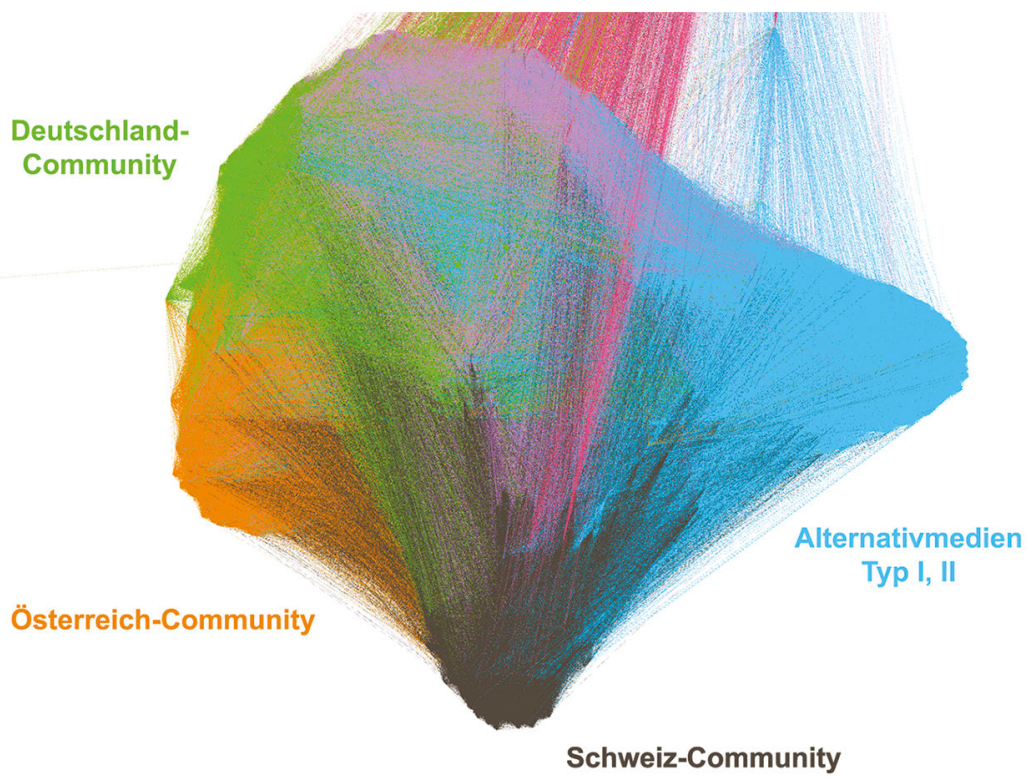

140 Verglichen mit dem Followernetzwerkalternativer Nachrichtenmedien ist die durchschnittliche Pfadlänge dieses Netzwerks deutlich höher. Im Schnitt existieren also sehr viel mehr Knoten zwischen zwei Knotenpunkten innerhalb des Netzwerks. Der Modularitätswert ist vergleichsweise höher, wonach die Verbindungen innerhalb der Communities dichter sind und weniger Anknüpfungen zu den weiteren Communities existieren. 
Auch in diesem Fall kann nicht von einer Echokammernbildung ausgegangen werden, vielmehr sind die Communities untereinander vernetzt. Dennoch lassen sich aus interpretativer Sicht deutliche Unterschiede zwischen den vier visualisierten Follower-Communites erkennen. Vor allem im Vergleich zum eben beschriebenen Follower-Netzwerk, das sich allein auf den Datensatz alternativer Nachrichtenmedien stützt, wird ausserdem noch klarer, dass alternative und professionelle Nachrichtenmedien unterschiedliche Publika ansprechen. Die Kennwerte der zentralen Knotenpunkte sind in Anhang $2 \mathrm{~b}$ aufgeführt.

An diesem Netzwerk fällt zunächst besonders die Tatsache auf, dass die drei Untersuchungsländer innerhalb der Communities noch stärker zum Ausdruck kommen. Insofern ist eine Unterscheidung in eine (Deutsch-)Schweizer, österreichische und deutsche Community möglich. Interessant ist hierbei vor allem, dass sich trotz der globalen Ausrichtung von Social-Media-Plattformen wie Twitter länderspezifische Communities herausbilden, die auch auf einen Binnendiskurs innerhalb der drei deutschsprachigen Länder hinweisen ${ }^{141}$. Die österreichische Community ist in orange visualisiert und nimmt 6,37\% des Gesamtnetzwerks ein. Unter den Nachrichtenmedien sind vor allem die Twitter-Auftritte von Der Standard (mit einem Eingangsgrad von 6'381), Der Falter (Eingangsgrad: 4'322) und Der Kurier (Eingangsgrad: 3'644) als starke Knotenpunkte innerhalb des Clusters zu identifizieren, oder aber Profil (Eingangsgrad: 3'867) und Die Presse (Eingangsgrad: 3'435). Eine noch grössere «Deutungsmacht» im Sinne von Knotenstärke weisen allerdings der Account der österreichischen Politikwissenschaftlerin Natascha Strobl (Eingangsgrad: 11'796) und des Journalisten Armin Wolf auf (Eingangsgrad: 11'580). Dies zeigt deutlich, dass vor allem die Personalisierung einen wesentlichen Stellenwert auf sozialen Netzwerken einnimmt. Neben den mittlerweile gesellschaftlich erwarteten Twitter-Accounts der Medienmarken sind es vor allem die jeweiligen Journalist_innen, die die sozialen Netzwerke bewirtschaften. So beispielsweise auch Kurier-Journalist Robert Misik (Eingangsgrad: 4'891), Politikwissenschaftlerin und Journalistin Corinna Milborn (Eingangsgrad: 5'454) oder die österreichische Journalistin und Autorin Ingrid Brodnig (Eingangsgrad: 4'798). Auch politische Akteur_innen sind auf der Plattform aktiv; nicht verwunderlich ist daher die Prominenz des Twitter-Kanals des österreichischen Bundespräsidenten Alexander Van der Bellen (Eingangsgrad: 6'820) innerhalb der Community und weiterer österreichischer Politiker_innen (z. B. des Bundeskanzlers Sebastian Kurz (Eingangsgrad: 9'635), des ehemaligen SPÖ-Politikers Christian Kern (Eingangsgrad: 4'558) oder des Grünen-Politikers Werner Kogler (Eingangsgrad: 4'056)). Auch österreichische alternative Nachrichtenmedien finden sich in der «Österreich-Community», darunter etwa Kontrast (Eingangsgrad: 2'738), ein

141 Dies soll aber nicht bedeuten, dass ausschliesslich z. B. Schweizer User_innen innerhalb des als «Schweiz-Community» bezeichneten Clusters vorzufinden sind. 
sozialdemokratisch positioniertes alternatives Nachrichtenmedium, und Mosaik (Eingangsgrad: 1'252), die beide Typ III der Typologie («Aufstand der Zivilgesellschaft»; vgl. Kapitel VII.2) zuzuordnen sind. In der gleichen Community - und entsprechend eine ähnliche Followerstruktur aufweisend - befindet sich das alternative Nachrichtenmedium Addendum mit seinem Twitter-Account daswasfehlt (Eingangsgrad: 2'904). Dieses entspricht typologisch betrachtet Alternativmedientyp IV «Die seriöse Alternative». Die in der österreichischen Community platzierten alternativen und professionellen Nachrichtenmedien sind aus einer politischen Perspektive vorwiegend links- oder mitte-linksorientiert.

Die angrenzende grüne Community (27,32\% des gesamten Netzwerks) vereint vor allem deutsche Nachrichtenmedien und weitere Akteur_innen aus Deutschland («Deutschland-Community»). Unter den Nachrichtenmedien sind in dieser Community am stärksten ZeitOnline (Eingangsgrad: 28'842), die deutsche Tagesschau (Eingangsgrad: 27'958), Spiegel Online (Eingangsgrad: 27'099), die Süddeutsche Zeitung (Eingangsgrad: 25'930), Die Welt (Eingangsgrad: 17'923), die FAZ (Eingangsgrad: 16'895), ZDF (Eingangsgrad: 16'354) und die Taz (Eingangsgrad: 20'144) vertreten. Neben die in den 1970er-Jahren gegründete Taz, die ob ihrer systemkritischen und linksalternativen Haltung auch als alternatives Nachrichtenmedium eingeordnet werden kann, reiht sich das Alternativmedium Volksverpetzer (Eingangsgrad: 8'201) ein, das - wie im vorigen Kapitel erläutert - sich selbst als Media-Watchdog und weniger als kontinuierliches Nachrichtenmedium positioniert. Ähnliches gilt für die Twitter-Auftritte der Korrektivseiten Correctiv.org (Eingangsgrad: 10'388) und BILDblog (Eingangsgrad: 12'866) wie auch das «seriöse Alternativmedium» (vgl. Typologie Kapitel VII.2) Krautreporter (Eingangsgrad: 7'761), die demnach das Publikum professioneller Leit- bzw. «Mainstreammedien» erreicht haben. Weitere Akteur_innen innerhalb des Clusters sind beispielsweise Greta Thunberg (Eingangsgrad: 27'433) und die Bewegung Fridays for Future (Eingangsgrad: 10'627), Die Grünen Deutschland (Eingangsgrad: 13'275) wie auch Satiriker Jan Böhmermann (Eingangsgrad: 28'388) oder das Satire-Magazin Titanic (Eingangsgrad: 14'708). Auch weitere deutsche politische Parteien wie Die Linke (Eingangsgrad: 9'275) oder die CDU (Eingangsgrad: 8'195) sind Teil der Community.

Die Schweiz-Community - als dritte «Ländercommunity» - ist im Netzwerk am unteren Teil in schwarz visualisiert und nimmt einen Anteil von 9,88\% ein. Der stärkste Knoten innerhalb dieses Clusters ist die NZZ mit einem Eingangsgrad von 15'732, weitere starke Knotenpunkte unter den professionellen Nachrichtenmedien sind SRF News (Eingangsgrad: 6'116), der Tages-Anzeiger (Eingangsgrad: 5'832), Watson (Eingangsgrad: 4'860), der Blick (Eingangsgrad: 3'813) oder auch die Wochenzeitung (Eingangsgrad: $3^{\prime} 672$ ). In hohem Masse innerhalb der Community vertreten sind - wie zu erwarten war - die persönlichen Accounts von Schweizer Journalist_innen, wie z. B. Fabian Eberhard (SonntagsBlick) (Eingangsgrad: $3^{\prime} 769$ ) oder Adrienne Fichter (Republik) (Eingangsgrad: 3'244). Ebenso sind in dem Clus- 
ter auch die Accounts der - als alternative Nachrichtenmedien einzuordnenden - Republik (Eingangsgrad: 4'934) und der Weltwoche (Eingangsgrad: 3'344) aufzufinden, die sich beide - wie bereits thematisiert - inhaltlich stark voneinander unterscheiden. Auch in diesem Netzwerk wird folglich offensichtlich, dass die Follower_innen von Schweizer alternativen Nachrichtenmedien auch mit Schweizer professionellen Leitmedien konfrontiert werden oder allenfalls unterschiedliche Medientypen abonniert haben ${ }^{142}$.

Die blaue Community nimmt 10,27\% des Gesamtnetzwerks ein und verdeutlicht die quantitative Präsenz des typologisch als Typ I beschriebenen Alternativmedientyps «Aufdecker der Mainstreamlügen» und des Typs II «Verschwörung und Spiritualität» (vgl. Kapitel VII.2). Starke Knoten innerhalb dieser Community sind vorwiegend deutsche alternative Nachrichtenmedien, wie Tichys Einblick (Eingangsgrad: 6'152) (wie auch Roland Tichys persönlicher Account selbst mit einem noch höheren Eingangsgrad von 8'801), Cicero (Eingangsgrad: 7'057), Die Achse des Guten (Eingangsgrad: 5'664), die Junge Freiheit (Eingangsgrad: 5'008) oder auch Russia Today Deutschland (Eingangsgrad: $4^{\prime} 268$ ), das COMPACT Magazin (Eingangsgrad: 3'840) und NachDenkSeiten (Eingangsgrad: 3'831). Einen schwächeren Knotenpunkt, aber dennoch einen Teil der Community, stellt das österreichische alternative Nachrichtenmedium Unzensuriert (Eingangsgrad: 1'129) dar. Jene Medien positionieren sich selbst als Opposition zum «Mainstream» dahingehend, dass sie diesen der Lügen oder als Teil von Verschwörungen bezichtigen (vgl. Kapitel VII.2). Aber nicht nur Nachrichtenmedien sind Teil der blau markierten Community. Wie bereits im Follower-Netzwerk der alternativen Nachrichtenmedien im vorigen Kapitel ersichtlich, reihen sich auch rechtspopulistische Parteien und Politiker_innen in diese Community ein. Dies ist nicht weiter verwunderlich, zumal sich alternative Nachrichtenmedien dieses Typs I in ihrer Berichterstattung häufig pro rechtsorientierte Parteien und deren Akteur_innen aussprechen. Neben der AfD (Eingangsgrad: 6'254) oder der AfD-Politikerin Alice Weidel (Eingangsgrad: 7'040) ist beispielsweise auch der Schweizer SVP-Politiker Roger Köppel (Eingangsgrad: 4'178) ein starker Knotenpunkt innerhalb der Community. Die Einordnung solcher Fälle ist besonders interessant, da diese aus netzwerkanalytischer Sicht nicht in den passenden Ländercommunities positioniert sind, sondern im länderübergreifenden Cluster, der aus politischer Sicht eine klare Rechtspositionierung repräsentiert. Die politische Gleichgesinnung scheint in diesem Fall ein stärkerer Faktor für die Zugehörigkeit einer Follower-Community zu sein als die Nationalität.

142 Was gleichzeitig auch einen entbündelten, emergenten Medienkonsum auf Digitalplattformen deutlich macht: Nutzer_innen verfügen demnach über ihr eigenes Medienmenü oder Medien- bzw. Newsrepertoire, das sich weniger auf einzelne Medienmarken, sondern vielmehr auf ein Bündel unterschiedlicher Marken bezieht (Schneider \& Eisenegger, 2019; Schwaiger, 2019). 


\section{VII.3.1.3 Gegenüberstellung der Follower-Netzwerke}

Die beiden vorgängig beschriebenen Follower-Netzwerke geben vor allem Aufschluss darüber, welche Nachrichtenmedien über ähnliche Publika im Sinne von Twitter-Abonnent_innen verfügen. Kritisch reflektiert werden muss an dieser Stelle, dass ein Twitter-Abonnement nicht unbedingt mit einer zustimmenden Haltung zwischen Follower_in und Akteur_in einhergehen muss, weshalb sich Kapitel VII.4 einem interpretativen Zugang der Netzwerke widmet. Dennoch geben die beiden illustrierten Netzwerke einen deskriptiven Überblick über die Publika respektive Communities deutschsprachiger Nachrichtenmedien. Lohnend ist die getrennte Betrachtung der beiden Netzwerke, um erstens den Fokus auf die Communities alternativer Nachrichtenmedien zu richten und zweitens die gesamte deutschsprachige Medienlandschaft - inklusive professioneller Nachrichtenmedien - zu untersuchen. Die Einteilung in Communities kann dabei auch - einem Repertoireansatz folgend - einen Anhaltspunkt bezüglich der individuellen Nachrichtenmenüs von Twitter-Nutzer_innen geben (z. B. Edgerly, 2015; Hasebrink \& Hepp, 2017; Rauchfleisch et al., 2020; Schmidt, Merten, Hasebrink, Petrich \& Rolfs, 2019; Schneider \& Eisenegger, 2018; Schneider \& Eisenegger, 2019). Bezogen auf das erste Follower-Netzwerk, das sich auf alternative Nachrichtenmedien konzentriert, kann demnach von drei Alternativmedien-Repertoiretypen auf Twitter ausgegangen werden. Die kleinste Community bezieht sich dabei auf die Rezipient_innen Schweizer Alternativmedien (und weiterer Schweizer professioneller Nachrichtenmedien), unabhängig davon, welche subjektive, «typologische» Ausrichtung diese haben. Die Community wird demnach stärker durch ihren Länderbezug definiert als durch ihre journalistische (und gegebenenfalls politische) Ausrichtung. Zwei Communities sind besonders prominent im Netzwerk: Einerseits jene Community, die neben professionellen Nachrichtenmedien auch politisch linksorientierte Akteur_innen (z. B. Fridays for Future), Korrektivseiten (z. B. Correctiv.org) und allenfalls alternative Nachrichtenmedien, die als Typ IV «Die seriöse Alternative» einzuordnen sind, abonniert hat. Andererseits die Community der alternativen Nachrichtenmedien des Typs I «Aufdecker der Mainstreamlügen» und des Typs II «Verschwörung und Spiritualität», die sich schliesslich beide häufig sehr ähnlichen Themen widmen, wenn auch in einer unterschiedlichen Rahmung. Ebenso befinden sich in diesem Cluster (oder Repertoire) rechtspopulistische Akteur_innen und Parteien, wie z. B. die AfD. Festzuhalten ist, dass mit Blick auf die Netzwerkstrukturen nicht von einer Echokammernbildung ausgegangen werden kann; vielmehr sind die drei Communities - zumindest strukturell - stark untereinander vernetzt. Die inhaltlichen Vernetzungen werden in Kapitel VII.4 interpretiert.

Die Analyse der Followerstrukturen unter Berücksichtigung sämtlicher alternativer wie auch professioneller Nachrichtenmedien bestätigte einerseits die Communities des reduzierten Follower-Netzwerks und zeigte weiter ein diffe- 
renziertes Bild der deutschsprachigen Nachrichten-Twittersphäre auf. Deutlich wurde, dass sich die Twitter-Nachrichtenrepertoires stark anhand der drei Untersuchungsländer strukturieren. So ist neben einer «Schweiz-Community» auch eine österreichische und deutsche Community ersichtlich. In allen drei Communities finden sich alternative Nachrichtenmedien aus ebendiesen Ländern, vor allem jene, die Typ III («Aufstand der Zivilgesellschaft») und Typ IV («Die seriöse Alternative») zuzuordnen sind. Auffallend ist auch in diesem Netzwerk die - aus quantitativer Sicht - grosse Community (10,27\%), die in ähnlicher Weise wie im vorgängig beschriebenen Netzwerk den Alternativmedien-Cluster «Aufdecker der Mainstreamlügen» repräsentiert, der auch vereinzelt - ob der ähnlichen thematischen Schwerpunkte - alternative Nachrichtenmedien des Typs II «Verschwörung und Spiritualität» beinhaltet. Im Vergleich zu den restlichen Communities ist hier auffällig, dass nicht nur Akteur_innen und Medien aus Deutschland vertreten sind, sondern auch aus Österreich und der (deutschsprachigen) Schweiz. Diese können eindeutig im rechten politischen Spektrum eingeordnet werden. Diese Community fasst entsprechend Follower_innen, die vorwiegend rechtsorientierte Politiker_innen und alternative Nachrichtenmedien abonnieren. Diese Medien und Akteur_innen positionieren sich klar gegen das «Establishment», das sich allerdings nicht auf einzelne Länder, sondern den gesamten deutschsprachigen Raum bezieht. Dieser Befund verdeutlicht, dass rechtspopulistische Akteur_innen länderüberschreitend miteinander vernetzt sind und entsprechend ein länderübergreifendes Publikum bedienen.

$\mathrm{Zu}$ Beginn des Kapitels VII.3.1 wurden Thesen aus den bisherigen empirischen Analysen in dieser Arbeit abgeleitet. Bezugnehmend auf die beiden Follower-Netzwerke lassen sich nun folgende Befunde festhalten: These 1 geht davon aus, dass sich (in diesem Fall Follower-)Communities rund um die vier Typen alternativer Nachrichtenmedien bilden. Diese These kann teilweise bestätigt werden. Beide analysierten Netzwerke zeigen deutlich, dass Followercommunities vor allem einen Länderbezug aufweisen. Follower_innen strukturieren sich demnach vorwiegend um Nachrichtenmedien und weitere Twitter-Akteur_innen aus den gleichen Ländern. Dies trifft auch auf alternative Nachrichtenmedien zu, vor allem jene des Typs III («Aufstand der Zivilgesellschaft) und des Typs IV («Die seriöse Alternative»). Allerdings zeigt sich in den Netzwerken auch - These 1 bestätigend -, dass alternative Nachrichtenmedien des Typs I («Aufdecker der Mainstreamlügen») und des Typs II («Verschwörung und Spiritualität») auch länderübergreifend hinsichtlich ihrer Followerstrukturen stark untereinander vernetzt sind. Auffallend ist weiter, dass es - aus netzwerkanalytischer Sicht - stärkere Überschneidungen zwischen Typ I und II wie auch Typ III und IV gibt. Diese Relationen basieren entweder auf einer ähnlichen politischen Werthaltung oder ähnlichen Themensetzung in der Berichterstattung. Detaillierte Ergebnisse hierzu werden in Kapitel VII.4 geschildert. 
These 2 geht davon aus, dass vor allem alternative Nachrichtenmedien des Typs I («Aufdecker der Mainstreamlügen») stark in den (Follower-)Netzwerken vertreten sind. Aus quantitativer Sicht nehmen die Communities, die diesem Alternativmedientyp entsprechen ${ }^{143}, 14,35 \%$ bezogen auf das Netzwerk alternativer Nachrichtenmedien und $10,27 \%$ bezogen auf das Netzwerk alternativer und professioneller Nachrichtenmedien ein. Die These ist somit bestätigt. Die restlichen drei Typen alternativer Nachrichtenmedien konnten hinsichtlich ihrer Followerstrukturen nicht als eigenständige Communities identifiziert werden, sondern sind Teil der Länder-Cluster.

These 3 bezieht sich auf die Vernetzung alternativer Nachrichtenmedien mit politischen Akteur_innen. Es wird angenommen, dass alternative Nachrichtenmedien mit politisch Gleichgesinnten vernetzt sind. Bezogen auf die Followercommunities konnten in den Netzwerken teilweise politische Cluster identifiziert werden, sowohl in den Ländercommunities als auch in der Community der «Alternativen Aufdecker» in beiden Netzwerken. Dabei sticht letztere Community heraus, die sich eindeutig um rechtsorientierte bis hin zu rechtspopulistische Twitter-Akteur_innen strukturiert. Bei den weiteren Communities hingegen, die einen deutlichen Länderbezug aufweisen, würde eine politische Einordnung zu weit greifen. Vielmehr handelt es sich bei den drei Ländercommunities um den medialen und politischen «Mainstream».

Die offen gehaltene These 4 kann nur ansatzweise durch die Analyse der beiden Follower-Netzwerke diskutiert werden. Angenommen wird hierbei, dass die Alternativmedientypen in unterschiedlicher Weise mit dem medialen und politischen «Mainstream» vernetzt sind. Dies ist kaum ausschliesslich anhand der Followerbeziehungen zu beurteilen, dennoch lässt sich feststellen, dass alternative Nachrichtenmedien der Typen I und II über ähnliche Followerbeziehungen wie politisch rechtsorientierte Twitter-Akteur_innen verfügen und vom medialen «Mainstream», also den professionellen Leitmedien, netzwerkanalytisch distanziert sind. Alternative Nachrichtenmedien der Typen III und IV hingegen befinden sich innerhalb ihrer Ländercommunities, in denen auch professionelle Informationsmedien der jeweiligen Länder platziert sind, wie auch politische Akteur_innen, die nicht in den rechtspopulistischen Cluster fallen. Insofern zeichnet sich - zumindest bezugnehmend auf die Followerstrukturen - ab, dass alternative Nachrichtenmedien in unterschiedlichem Ausmass vom medialen und politischen «Mainstream» distanziert sind.

Die in diesem Kapitel beschriebenen Follower-Netzwerke geben Aufschluss über die Verteilung der Publika alternativer wie auch professioneller Nachrichtenmedien aus Deutschland, Österreich und der deutschsprachigen Schweiz. Die Communities können dabei auch als «Newsrepertoires» definiert werden, wo-

143 Obwohl auch alternative Nachrichtenmedien des Typs II Teil dieser Communities sind. 
nach einzelne Cluster über ein bestimmtes Set an genutzten Nachrichten verfügen. Im folgenden Kapitel werden weitere Netzwerkanalysen basierend auf den beiden Datensätzen analysiert und beschrieben. Hierbei handelt es sich um Retweet-Netzwerke, die nicht auf den Followerbeziehungen, sondern auf den gegenseitigen Bezugnahmen zwischen Akteur_innen über Retweets beruhen.

\section{VII.3.2 Retweet-Netzwerkstrukturen}

Die in diesem Kapitel beschriebenen Retweet-Netzwerke haben zum Ziel, Relationen zwischen Twitter-Akteur_innen (mit einer entsprechenden Fokussierung auf alternative Nachrichtenmedien) aufzudecken. Hierfür eignet sich die netzwerkanalytische Betrachtung von Retweets in besonderem Masse, da die Twitter-Funktion des Beitrag-Teilens erstens sehr stark in der Twittersphäre genutzt wird und zweitens einen quantitativen Überblick darüber gibt, welche Akteur_innen miteinander in Verbindung stehen, also aktiv auf Inhalte anderer verweisen. Diese Art von Akteursbeziehungen sind ob ihrer Inhalte ausserdem mit «Sinn» gefüllt und geben - aus einer relational-soziologischen Perspektive - weiter Aufschluss über die Sinnstrukturen innerhalb des Netzwerks. Dieser interpretativen Analyse widmet sich Kapitel VII.4, nachdem folgend eine deskriptive Analyse der Retweetstrukturen alternativer (vgl. Kapitel VII.3.2.1) sowie alternativer und professioneller Nachrichtenmedien (vgl. Kapitel VII.3.2.2) durchgeführt wird.

\section{VII.3.2.1 Retweet-Netzwerk alternativer Nachrichtenmedien}

Auch die folgenden Retweet-Netzwerkanalysen basieren auf den zwei Datensätzen, die einerseits Tweets über alternative Nachrichtenmedien und andererseits Tweets über alternative und professionelle Nachrichtenmedien aus Deutschland, Österreich und der deutschsprachigen Schweiz beinhalten. Hierfür wurden aus den zugrundeliegenden Datensätzen zunächst sämtliche Tweets extrahiert, die retweetet wurden. Aus diesen Subsamples wurden sämtliche Twitter-Accounts jener Akteur_innen, die einen Tweet abgesendet, und jenen, die diesen retweetet haben, in separaten Datensätzen abgespeichert. In diesem Subkapitel wird das Retweet-Netzwerk alternativer Nachrichtenmedien veranschaulicht (vgl. Anhang 2c).

Insgesamt wurden 109'802 Retweet-Relationen zwischen Akteur_innen identifiziert («Kanten»), ausgehend von 34'664 Knotenpunkten, also Twitter-Accounts. Das Netzwerk weist eine mittlere Pfadlänge von 9,005 und einen Modularitätswert von 0,526 auf, insgesamt wurden mittels Louvain-Algorithmus 474 Communities identifiziert - weshalb aufgrund dieser hohen Zahl die Kantendichte gegen $o$ tendiert. Allerdings weisen nur vier Communities einen prozentuellen Anteil (auf das Gesamtnetzwerk bezogen) von über $10 \%$ auf. Die Beschreibung der Communities beschränkt sich folgend auf die quantitativ grössten Communities mit einem jeweiligen prozentualen Anteil von über $3 \%$. In Abbildung 22 wird das 
mittels Gephi visualisierte Retweet-Netzwerk für alternative Nachrichtenmedien dargestellt. Jeweils farblich markiert sind wiederum die einzelnen Communities.

\section{Abbildung 22: Twitter-Retweet-Netzwerk alternativer Nachrichtenmedien (Eigene Darstellung)}

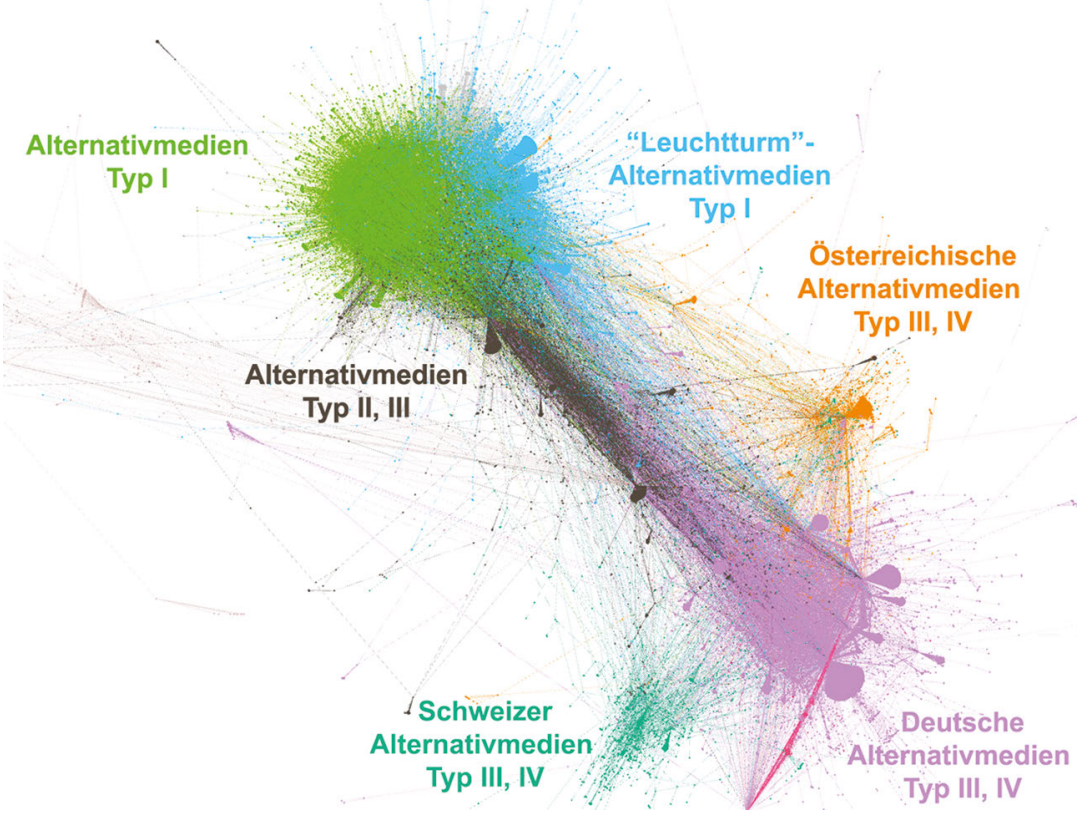

Verglichen mit den im vorigen Kapitel beschriebenen Follower-Netzwerken wird hier deutlich, dass die Communities stärker voneinander polarisiert sind. So finden sich vor allem zwei Gruppen von Communities (dominiert durch die Communities in grün/türkis und lila), die voneinander distanziert und vereinzelt durch einzelne Twitter-Accounts miteinander verbunden sind. Die mit 39,32 \% grösste Community des Netzwerks ist in Abbildung 22 lila markiert. Vergleichbar ist die Community mit der ebenfalls lila markierten Community des Follower-Netzwerks alternativer Nachrichtenmedien (vgl. Kapitel VII.3.1.1), in der alternative Nachrichtenmedien des Typs IV («Die seriöse Alternative») inkludiert sind. Bezugnehmend auf die Community sind innerhalb des Retweet-Netzwerks z. B. Krautreporter mit einem Eingangsgrad von 304 sowie Korrektivseiten und Media-Watchdogs, darunter als stärkster Knoten in der Community vertreten der Twitter-Account von Volksverpetzer (Eingangsgrad: 6'207), der BILDblog (Eingangsgrad: 2'704) oder Uebermedien (Eingangsgrad: 1'546), vertreten. Teilweise sind zudem Alternativmedien des Typs III Teil der Community, so beispielsweise Lobby Control (Eingangsgrad: 886). Der Cluster besteht demnach aus alternativen Nachrichtenmedien, die politisch am 
linken Spektrum einzuordnen sind. Zumal sie allesamt Teil einer Community sind, werden ihre Beiträge häufig von ähnlichen Twitter-Akteur_innen retweetet, bzw. retweeten sie sich allenfalls sogar gegenseitig.

Ebenso auf der rechten Seite des Netzwerks vertreten - und über einzelne Verbindungen mit dem eben beschriebenen Cluster verbunden (lila) - ist die orange markierte Community mit einem Anteil von 4,37\% bezogen auf das gesamte Netzwerk. In der Community sind Twitter-Accounts österreichischer alternativer Nachrichtenmedien vorhanden, die - ähnlich dem Follower-Netzwerk - unterschiedlichen Typen alternativer Nachrichtenmedien angehören, darunter vor allem Typ III und Typ IV. Dabei ist der stärkste Knotenpunkt mit einem Eingangsgrad von 1'032 der Account von Kontrast.at (Typ III), gefolgt von Addendum (Twitter-Account: daswasfehlt; Typ IV) mit einem Eingangsgrad von 150 sowie Erstaunlich.at (Typ I; Eingangsgrad: 52). Wie in Abbildung 22 visualisiert, ist die Community stärker zerstreut als z. B. die Community der «linken alternativen Nachrichtenmedien» (lila). Dies zeigt sich beispielsweise am Knotenpunkt von Addendum (daswasfehlt), der als Verbindungsglied zu Communities auf der links positionierten Seite des Netzwerks gesehen werden kann. Darüber hinaus sind in der Community weitere österreichische Akteur_innen zu finden, wie z. B. die Journalisten Robert Misik (Eingangsgrad: 25) und Armin Wolf (Eingangsgrad: 21). Die Community lässt erkennen, dass Retweets vor allem auf der Länderzugehörigkeit der Akteur_innen beruhen.

Ein ähnliches Bild ergibt sich bei der - in türkis markierten - Community, die einen Anteil von 3,85\% bezogen auf das Gesamtnetzwerk und einen Schweiz-Fokus aufweist. Vertreten sind hier unter den alternativen Nachrichtenmedien vor allem der Twitter-Account der Schweizer Republik (Eingangsgrad: 213) wie auch des Nachrichtenmediums Das Lamm (Account: ZwitscherLamm; Eingangsgrad: 16) - beides Medien, die Typ IV zuzuordnen sind («Die seriöse Alternative»). Ebenso Teil der Community sind weitere Schweizer Akteur_innen, z. B. Reto Knutti (Professor für Klimaphysik an der ETH Zürich; Eingangsgrad: 213), Netzaktivistin Jolanda Spiess-Hegglein (Eingangsgrad: 74) oder die Republik-Journalist_innen Elia Blülle (Eingangsgrad: 51) und Adrienne Fichter (Eingangsgrad: 29). Abgesehen von Schweizer Twitter-Accounts befindet sich aber auch das österreichische Nachrichtenmedium Dossier innerhalb der Community (Eingangsgrad: 40). Obwohl es sich um ein österreichisches Alternativmedium handelt, verwundert die Positionierung in diesem Cluster nicht - so wäre das Alternativmedium auch als Typ IV zu klassifizieren, wie auch die Schweizer Republik oder Das Lamm.

Die drei eben beschriebenen Communities, darunter die «deutschen politisch links orientierten alternativen Nachrichtenmedien» sowie die «Schweizer» und «österreichischen» alternativen Nachrichtenmedien, bilden somit gesamthaft den grafisch rechts unten positionierten Teil des Netzwerks. Sie sind untereinander eng miteinander verzahnt und umso stärker von den Communities im oberen 
linken Bereich des Netzwerks distanziert. Die grösste Community in diesem Bereich, mit einem Anteil von 14,86\% bezogen auf das Gesamtnetzwerk, ist in Abbildung 22 in grün gekennzeichnet. Die Community charakterisiert sich durch alternative Nachrichtenmedien des Typs I («Aufdecker der Mainstreamlügen»). Besonders starke Knotenpunkte sind hier der Twitter-Kanal von Journalistenwatch mit einem Eingangsgrad von 1'257, Hartes Geld (Eingangsgrad: 526), Unzensuriert (Eingangsgrad: 442), Pressecop24.com (Eingangsgrad: 380) oder Politikstube (Eingangsgrad: 259). Ebenso starke Akteur_innen innerhalb dieses Clusters stellen Twitter-Accounts dar, die nicht auf eine konkrete Website verweisen, aber offenbar deutungsmächtige Figuren in diesem Kreis sind, z. B. burger_ein ${ }^{144}$ (Eingangsgrad: 1'475), wach_jetzt_auf(Eingangsgrad:1'139) oder deutsch365 (Eingangsgrad: 737). Diese Medien und Akteur_innen haben allesamt gemein, dass sie ihre Opposition zum «Mainstream» (wobei dieser Begriff selbst in diffamierender Art und Weise gebraucht wird) durch starke Kritik und Verleumdung politischer Akteur_innen oder medialer Eliten (Leitmedien) einnehmen. Dabei zeigte sich bereits im Rahmen der typologischen Untersuchung (vgl. Forschungsfrage 2, Kapitel VII.2), dass rechtspopulistische Äusserungen befürwortet oder selbst publiziert werden, während politisch links oder mittig positionierte Akteur_innen diffamiert werden. Dies wird auch an politischen Knotenpunkten innerhalb des Clusters offensichtlich; so ist beispielsweise die ehemalige CDU-Politikerin und AfD-Unterstützerin Erika Steinbach stark in der Community vertreten (Eingangsgrad: 499), oder auch der Account AfD_Support (Eingangsgrad: 152). Es handelt sich vor allem um Twitter-Accounts respektive alternative Nachrichtenmedien aus Deutschland, aber auch hier sind Tendenzen einer länderübergreifenden Vernetzung feststellbar, was z. B. durch die Accounts von Wochenblick (Eingangsgrad: 552) und Unzensuriert (Eingangsgrad: 442) deutlich wird (beide haben ihren Ursprung in Österreich).

Eng verzahnt mit der eben beschriebenen, grün markierten Community ist die in blau visualisierte Community, mit einem fast gleich grossen Anteil von 14,35 \%. Gesamthaft bilden sie eine Art sozialen Kreis. Dies ist nicht verwunderlich, wenn die dort positionierten Akteur_innen genauer betrachtet werden. Es existieren starke inhaltliche Überschneidungen; auch in diesem Fall handelt es sich um alternative Nachrichtenmedien, die am besten dem Typ I zugeordnet werden können. Die stärksten Knotenpunkte bilden die Twitter-Accounts von Tichys Einblick (Eingangsgrad: 3'872) wie auch der Privataccount von Roland Tichy (Eingangs-

\footnotetext{
144 Der Twitter-Account burger_ein war zum Zeitpunkt der Auswertung der Netzwerkanalyse (02.08.2020) nicht mehr auf Twitter verfügbar. Dieser Account wurde demnach entweder von dem/der Betreiber_in selbst gelöscht, oder-durchaus denkbar-von Twitter gesperrt, da gegen die Richtlinien verstossen wurde. Mehrheitlich wurde im Rahmen der Analyse ersichtlich, dass vor allem Accounts oder Websites stark rechtspopulistischer Akteur_innen gelöscht wurden, da sie beispielsweise volksverhetzende Inhalte publiziert haben.
} 
grad: 3'302), Cicero Online (Eingangsgrad: 1'117) und Junge Freiheit (Eingangsgrad: 926). Es handelt sich hierbei um deutsche alternative Nachrichtenmedien, die auch über ihre Websites über sehr hohe Reichweiten verfügen (vgl. Forschungsfrage 1, Kapitel VII.1). Darüber hinaus sind in der Community Akteur_innen aus dem weiteren deutschsprachigen Raum vorhanden, beispielsweise der SVP-Politiker und Chefredakteur von Die Weltwoche Roger Köppel (Eingangsgrad:360), der ebenso als starker Knotenpunkt einzuordnen ist, wie auch der Twitter-Account dieses Nachrichtenmediums (Eingangsgrad: 65). Berechtigterweise stellt sich die Frage, warum die «grüne» und «blaue» Community als getrennte Communities resultieren. Aus inhaltlich-interpretativer Sicht der angesprochenen alternativen Nachrichtenmedien lässt sich ein wesentlicher Unterschied feststellen. Die in der blauen Community positionierten Alternativmedien unterscheiden sich nicht nur hinsichtlich ihrer Reichweite von der grün visualisierten Community, sondern auch bezugnehmend auf ihre Darstellungsform. So liess sich bereits im Zuge der typologischen Untersuchung feststellen, dass Typ I unter anderem alternative Nachrichtenmedien impliziert, die hochgradig professionalisiert sind. Dies wird nicht nur anhand der redaktionellen (eigentlich «klassisch journalistischen») Strukturen deutlich, sondern vor allem auch hinsichtlich des Layouts. Insofern sind jene alternativen Nachrichtenmedien, die in der blauen Community positioniert sind, rein optisch kaum von traditionellen Nachrichtenmedien unterscheidbar. Sie positionieren sich zwar eindeutig gegen den «Mainstream», also das politische und mediale Establishment, ihre alternative Grundhaltung wird aber rein optisch nicht deutlich. Alternative Nachrichtenmedien dieser Art könnten somit gewissermassen als «Leuchtturm-Alternativmedien» bezeichnet werden, die zwar alternative Positionen vertreten, aber dennoch in gewisser Weise den per Selbstbeschreibung kritisierten Mainstream erreicht haben - wenn auch in einer anderen Form, nämlich bezugnehmend auf ihre Darstellungsform und ggf. höhere Reichweiten im Vergleich zu alternativen Nachrichtenmedien, die sich am Ende des «Longtails» der Öffentlichkeit befinden.

Abschliessend für dieses Netzwerk wird die in dunklem Grau visualisierte Community mit einem Anteil von 10,38 \% fokussiert. Dieser Cluster ist insofern interessant, als er verbindend zwischen den «linken» und «rechten» Communities agiert und alternative Nachrichtenmedien der Typen II und III beinhaltet. Besonders starke Knotenpunkte sind in der grauen Community beispielsweise Russia Today Deutsch (Eingangsgrad: 2'156), Team KenFM (Eingangsgrad: 559), Sputnik Deutschland (Eingangsgrad: 336), cashkurs (Eingangsgrad: 198), weltnetzTV (Eingangsgrad: 76) oder das Contra Magazin (Eingangsgrad: 51). Diesen Seiten ist gemein, dass sie unter den Typ II «Verschwörung und Spiritualität» subsumiert werden können. Sie weisen demnach häufig einen verschwörungstheoretischen Charakter in ihren publizierten Inhalten auf und gehen von einer grösseren Elitenverschwörung aus, die hinter gesellschaftlichen Einzelereignissen verborgen 
ist. Ein besonders starker Akteur in diesem Cluster ist - zum Narrativ dieser alternativen Nachrichtenmedien passend - der Schweizer Historiker Daniele Ganser (Eingangsgrad: 122), der vor allem in verschwörungstheoretischen Kreisen Popularität erlangte ${ }^{145}$. Die Akteur_innen innerhalb der Community verdeutlichen die bereits in der typologischen Untersuchung alternativer Nachrichtenmedien (vgl. Kapitel VII.2) erörterte Unterscheidung zwischen Typ I («Aufdecker der Mainstreamlügen») und Typ II («Verschwörung und Spiritualität»): Sind es inhaltlich nur feine Unterschiede, die die beiden Typen voneinander trennen, zeigt die netzwerkanalytische Untersuchung, dass diese durchaus von Relevanz sind. Interessant ist zudem die Positionierung der Community zwischen den politisch eher links und eher rechst zu verordnenden Bubbles. Verdeutlicht wird diese ambivalente Position durch weitere alternative Nachrichtenmedien, die Teil des Clusters sind. So beispielsweise die Junge Welt (Eingangsgrad: 1'023) oder Rote Fahne News (Eingangsgrad: 89), die dem Typ III («Aufstand der Zivilgesellschaft») am besten zuzuordnen sind. Typ III ist wie auch Typ II elitenkritisch, allerdings aus einer sozialdemokratischen, antikapitalistischen Perspektive. Dennoch scheint es inhaltliche gemeinsame Nenner zu geben, die eine gemeinsame Verortung in der Community erklären. Dieser Frage wird vor allem in Kapitel VII.4 nachgegangen, in dem eine interpretative Auswertung der Netzwerkstrukturen erfolgt. Dennoch ist bereits an dieser Stelle zu vermuten - die Annahmen der typologischen Untersuchung (Forschungsfrage 2) bestätigend -, dass Typ II «Verschwörung und Spiritualität» vorrangig nicht politisch geprägt oder gefärbt ist. Während sich bei Typ I eine deutliche politische Rechtspositionierung dahingehend äussert, dass «linke Politik» diskreditiert wird, und Typ III und Typ IV eher am linken politischen Spektrum einzuordnen sind, scheint Typ II keine eindeutige politische Positionierung zu besitzen ${ }^{146}$. Im Zentrum steht vielmehr der Gedanke einer Elitenverschwörung, unabhängig von der politischen Couleur.

Das eben beschriebene Netzwerk bestätigt die typologische Unterscheidung alternativer Nachrichtenmedien. Es sind vor allem Typ I («Aufdecker der Mainstreamlügen»), auf der linken oberen Seite der Abbildung 22, und Typ IV («Die seriöse Alternative»), auf der rechten unteren Seite, die stark voneinander polarisiert erscheinen. Typ II («Verschwörung und Spiritualität») nimmt eine zwischen den Communities verbindende Rolle ein, während Typ III («Aufstand der Zivilgesellschaft») wohl auch aufgrund seiner vergleichsweise quantitativen Minderheit nicht eindeutig einer Community zuordenbar ist. Im folgenden Kapitel erfolgt (äquivalent zu den Follower-Community-Analysen) eine netzwerkanalytische Be-

145 Z. B. https://www.woz.ch/-768a (Stand: 03.08.2020)

146 Auch in diesem Fall eine rein idealtypische Annahme. Jede typologische Kategorisierung impliziert Mischtypen und «Ausreisser». 
trachtung von Retweetstrukturen bezugnehmend auf alternative wie auch professionelle Nachrichtenmedien.

\section{VII.3.2.2 Retweet-Netzwerk alternativer und professioneller Nachrichtenmedien}

Das in Abbildung 23 visualisierte Netzwerk beruht auf dem Gesamtdatensatz alternativer und professioneller Nachrichtenmedien. Insgesamt konnten darin 402'417 Retweet-Beziehungen («Kanten») von 121'187 Twitter-Accounts («Knoten») identifiziert werden. Darunter wurden mittels Louvain-Algorithmus 4'173 Communities identifiziert (Modularitätswert: 0,626 ), die mittlere Pfadlänge beträgt 7,605, die Kantendichte strebt gegen 0 , wonach gesamt betrachtet die einzelnen Knoten nur lose miteinander in Verbindung stehen. In der Abbildung wurden für eine bessere Übersicht jene Knotenpunkte fokussiert, die einen höheren Grad als 2 aufweisen, also mehr als zwei Verbindungen zu weiteren Akteur_innen haben. Dies traf für 51'799 Knoten zu (336'406 Kanten). Die Communities wurden wiederum farblich markiert, davon werden die sechs grössten Communities bezogen auf das Gesamtnetzwerk genauer beschrieben. Die Kennwerte der zentralen Knotenpunkte sind in Anhang $2 \mathrm{~d}$ aufgeführt.

Abbildung 23: Twitter-Retweet-Netzwerk alternativer und professioneller

Nachrichtenmedien (Eigene Darstellung) 
Einige bereits im vorigen Subkapitel beschriebene Communities finden sich auch im Gesamtnetzwerk wieder und werden durch weitere Communities der professionellen Nachrichtenmedien ergänzt. Somit wird auf einen ersten deskriptiven Blick ersichtlich, dass alternative und professionelle Nachrichtenmedien, bezogen auf ihre Retweetstrukturen, unabhängiger voneinander in Communities positioniert sind. Dies steht in Kontrast zu den Followercommunities (vgl. Kapitel VII.3.1.2), bei denen stärkere Überschneidungen zwischen den Abonnent_innen alternativer und professioneller Nachrichtenmedien identifiziert wurden. Die grösste Community mit einem Anteil von 22,87\% innerhalb des in Abbildung 23 dargestellten Netzwerks ist in lila markiert und repräsentiert vorwiegend den «Deutschen Mainstream» und beinhaltet fallweise auch alternative Nachrichtenmedien der Typen II und IV. Es handelt sich demnach um einen Cluster, der sich rund um deutsche, vorwiegend professionelle Nachrichtenmedien strukturiert - dies allerdings mit Ausnahmen, die es genauer zu interpretieren gilt (vgl. Kapitel VII.4). Der stärkste Knotenpunkt in diesem Cluster ist Spiegel Online (Eingangsgrad: 2'908), gefolgt vom Tagesspiegel (Eingangsgrad: 1'385), der Süddeutschen Zeitung (Eingangsgrad: 1'336) und weiteren professionellen Nachrichtenmedien. Neben diesen klassischen deutschen Leitmedien reiht sich auch die Taz (Eingangsgrad: 688) ein, die zu Gründungszeiten als alternatives Nachrichtenmedium antizipiert wurde, aber offenbar den «Mainstream» hinsichtlich Reichweite und Darstellungsform erreicht hat. Ebenso befinden sich die Twitter-Accounts von Übermedien (Eingangsgrad: 664) und Volksverpetzer (Eingangsgrad: 168) - bereits diskutiert als Medien für den medialen Metadiskurs («Watchdogs», Typ IV) - innerhalb des Clusters. Zudem sind auch Alternativmedien des Typs II Teil der Community, so beispielsweise Team KenFM (Eingangsgrad: 323), oder die NachDenkSeiten (Eingangsgrad: 243).

Eine weitere deutsche Community ist in Abbildung 23 in dunklem Grau zentral neben der eben beschriebenen Community platziert, weist aber einen deutlich geringeren Anteil von 6,78 \% auf. Aus rein deskriptiver Perspektive lassen sich kaum Unterschiede zum grünen Cluster feststellen, obschon Boulevardmedien einen höheren Stellenwert einnehmen. Die stärksten Knotenpunkte bilden hier das deutsche Boulevardmedium Die BILD (Eingangsgrad: 4'351), Der Spiegel (Eingangsgrad: 1'366), Focus Online (Eingangsgrad:1'010) wie auch weitere Accounts der BILD (z.B. BILD Promis, Eingangsgrad: 52) oder TAG24 Berlin (Eingangsgrad: 64).

Am weitesten im Netzwerk von der deutschen, lila visualisierten Community entfernt ist jene, die in grün visualisiert ist. Dies entspricht im Grossen und Ganzen der grünen Community im Retweet-Netzwerk alternativer Nachrichtenmedien, wie im vorigen Subkapitel erörtert. Dennoch scheinen die beiden Communities in diesem Netzwerk noch enger miteinander verzahnt zu sein - nämlich über mehrere weitere Cluster - als im vorgängig beschriebenen. Die in Abbildung 23 visualisierte grüne Community verzeichnet den zweitgrössten Anteil bezogen 
auf das Gesamtnetzwerk mit 10,86\% und beinhaltet vor allem Alternativmedien des Typs I. Der stärkste Knotenpunkt ist der Twitter-Account von Roland Tichy mit einem Eingangsgrad von 3'300; nicht verwunderlich zählt auch das Nachrichtenmedium Tichys Einblick zu den Top-5-Knotenpunkten dieser Community (Eingangsgrad: 1'818). Weitere alternative Nachrichtenmedien wie Hartes Geld (Eingangsgrad: 1'993), Journalistenwatch (Eingangsgrad: 1'150) oder die Junge Freiheit (Eingangsgrad: 924) reihen sich in diese Community ein. Typologisch zuzuordnen sind diese Medien - wie bereits erörtert - dem Typ I alternativer Nachrichtenmedien («Aufdecker der Mainstreamlügen»). Weitere deutungsmächtige Akteur_innen innerhalb der Community umfassen den ehemaligen deutschen Präsidenten des Bundesamtes für Verfassungsschutz und CDU-Politiker Hans-Georg Maaßen Eingangsgrad: 2'285), AfD-Politikerin Erika Steinbach (Eingangsgrad: 1'493) oder Roger Köppel (Eingangsgrad: 1'082) wie auch im vorigen Subkapitel bereits erwähnte Twitter-Accounts, die dem Narrativ der alternativen Nachrichtenmedien des Typs I entsprechen. Diesbezüglich seien beispielsweise deutsch365 (Eingangsgrad: 1'061), Einzelfallinfos ${ }^{147}$ (Eingangsgrad: 613), wach_jetzt_auf (Eingangsgrad: 609) oder Pressecop24com (Eingangsgrad: 343) genannt. Zwar ist der Cluster von deutschen alternativen Nachrichtenmedien dominiert, doch finden sich auch weitere Nachrichtenmedien aus dem deutschsprachigen Raum in der Community, wie etwa das österreichische Unzensuriert (Eingangsgrad: 431). Dies bestätigt erneut die bereits mehrmals belegte These, dass vor allem Nachrichtenmedien des Typs I länderübergreifend - sei es über Follower- oder Retweetstrukturen - eng miteinander verzahnt sind. Auch finden sich das österreichische Boulevardmedium Kronen Zeitung (Eingangsgrad: 700) oder der Account BILD Politik (Eingangsgrad: 709) innerhalb der Community von vorwiegend Alternativmedien des Typs I und nicht im Cluster der österreichischen Medien (orange markiert). Dies ist vor allem für die nachfolgende interpretative Analyse der Netzwerkrelationen von Interesse, in der u. a. eruiert werden soll, inwiefern Boulevardmedien mit alternativen Nachrichtenmedien in Verbindung stehen können (vgl. Kapitel VII.4).

Die drittgrösste Community in diesem Retweet-Netzwerk mit einem Anteil von 10,26\% ist in Abbildung 23 in blau gekennzeichnet. Der Cluster kann als «Schweiz-Community» bezeichnet werden; zu den starken Knotenpunkten zählen demnach vor allem Schweizer Leitmedien im Sinne von professionellen Nach-

147 Der Twitter-Account Einzelfallinfos beschreibt beispielsweise in seiner Profilinformation: «Meldungen zu Straftaten durch Flüchtlinge \& (mutmaßliche) Migranten» (https://twitter.com/ Einzelfallinfos Stand: 04.08.2020). Das Wort «Einzelfall» wird hier in einer ironischen Weise genutzt, was für diesen Alternativmedientyp I nicht unüblich ist. Dabei wird unmissverständlich aufdieBerichterstattungprofessioneller «Mainstreammedien»verwiesen, die Delikteausgehend von Migrant_innen so darstellen würden, dass nicht alle Personen mit Migrationshintergrund als Straftäter_innen diskreditiert würden. Dies wird von alternativen Medien dieses Typs I kritisiert, wobei im selben Zuge vor Straftaten ausgehend z. B. von Flüchtlingen gewarnt wird. 
richtenmedien. Den höchsten Eingangsgrad (also eingehende Verbindungen in Form von Retweets) mit einem Wert von I'985 weist der Twitter-Kanal SRF News auf, gefolgt vom Tages-Anzeiger (Eingangsgrad: 541), NZZ am Sonntag (Eingangsgrad: 496), Blick (Eingangsgrad: 380), Watson (Eingangsgrad: 295) und der Wochenzeitung (Eingangsgrad: 216). Es handelt sich dabei um unterschiedliche Medientypen, vom öffentlich-rechtlichen Rundfunk über Qualitätsmedien bis hin zu Boulevardmedien. Aber auch abseits des «Mainstreams» sind alternative Schweizer Nachrichtenmedien Teil des Clusters, darunter - wie zu erwarten - die Republik (Eingangsgrad: 86) oder (wenn auch mit einem sehr geringen Eingangsgrad von 3) der Twitter-Account von Das Lamm - beide typologisch als Typ IV («Die seriöse Alternative») einzuordnen. Neben Nachrichtenmedien sammelt der Cluster die Accounts von Akteur_innen des öffentlichen Lebens in der Schweiz. Besonders deutungsmächtig i. S. v. starken Knotenpunkten sind hier der ETH-Professor Reto Knutti (Eingangsgrad: 482), der sich vor allem mit Klimafragen auseinandersetzt, die Schweizer Netzaktivistin Jolanda Spiess-Hegglin (Eingangsgrad: 206) und die Twitter-Accounts Schweizer Politischer Parteien, wie beispielsweise der Liberalen (FDP; Eingangsgrad: 119), der Grünliberalen (Eingangsgrad: 91) oder der Sozialdemokratischen Partei (SP; Eingangsgrad: 67).

Das österreichische Pendant zur eben beschriebenen «Schweiz-Community» ist in orange visualisiert und besitzt einen Anteil von 5,35\% bezogen auf das Gesamtnetzwerk. Die Community beinhaltet österreichische professionelle und vereinzelt auch alternative Nachrichtenmedien. $\mathrm{Zu}$ den stärksten Knotenpunkten zählen die Twitter-Auftritte von Der Standard (Eingangsgrad: 1'472), Die Presse (Eingangsgrad: 366), dem Tech-Magazin futurzone (Eingangsgrad: 270) oder Der Kurier (Eingangsgrad: 180). Auch das sozialdemokratische alternative Nachrichtenmedium (Typ III) Kontrast (Eingangsgrad: 90) ist Teil des Clusters. Wie nach den bisherigen Netzwerkanalysen zu erwarten war, zählen auch österreichische Akteur_innen wie der Journalist Armin Wolf (Eingangsgrad: 756), Falter-Journalist Florian Klenk (Eingangsgrad: 381) oder Rechtsextremismus-Expertin Natascha Strobl (Eingangsgrad: 255) wie auch der Twitter-Account der Sozialdemokratischen Partei (SPÖ; Eingangsgrad: 76) zur «Österreich-Community».

Wie der vorgängig als «deutsche Community» beschriebene Cluster in grau, nimmt auch die pinke Community eine Sonderstellung ein und ist nicht eindeutig klassifizierbar. Mit einem Anteil von 8,18 \% weist sie zudem einen geringen Anteil bezogen auf das Gesamtnetzwerk auf. Stärkster Knotenpunkt ist die Schweizer NZZ (Eingangsgrad: 5'650), gefolgt vom deutschen Magazin Stern.de (Eingangsgrad: 3'208). Bei den weiteren Akteur_innen innerhalb der Community handelt es sich vorwiegend um Privataccounts von Personen, die in- oder ausserhalb Deutschlands für Katalonien bzw. die katalonische Regierung tätig sind. Insofern lässt sich diese Community als die internationalste Community beschreiben, dominiert von den Nachrichtenmedien NZZ und Stern.de. 
Gesamthaft offenbart das Netzwerk eine enge Verzahnung zwischen Communities, wobei die aus politisch-normativer Sicht distanziertesten auch im Netzwerk am weitesten voneinander entfernt sind. Dennoch sind sie über weitere Communities verbunden, weshalb aus deskriptiver Sicht nicht von einer Echokammernbildung gesprochen würde. Inwiefern die Communities inhaltlich voneinander distanziert oder polarisiert sind, wird im Rahmen der qualitativen Inhaltsanalyse in Kapitel VII.4 erörtert. Davor werden jedoch die beiden Retweet-Netzwerke dieses und des vorangegangenen Sub-Kapitels abschliessend gegenübergestellt.

\section{VII.3.2.3 Gegenüberstellung der Retweet-Netzwerke}

Die beiden Retweet-Netzwerke weisen bezogen auf ihre Communities starke Ähnlichkeiten auf; rein visuell treten gleichzeitig deutliche Unterschiede zu Tage. Insofern ist es durchaus ergebnisträchtig, einen getrennten Blick auf die beiden Netzwerke zu werfen. So zeigt sich bezugnehmend auf das Retweet-Netzwerk, das ausschliesslich auf Datenbasis alternativer Nachrichtenmedien entstand (vgl. Kapitel VII.3.2.1), dass die Retweet-Communities stärker voneinander distanziert sind, was auf eine inhaltliche Polarisierung hinweist. Aus deskriptiver Sicht wird deutlich, dass alternative Nachrichtenmedien eines Typs häufiger untereinander Beiträge retweeten, oder ihre Tweets von ähnlichen Twitter-Akteur_innen retweetet werden. Im Retweet-Netzwerk alternativer Nachrichtenmedien entstehen so zwei grössere Cluster, die vor allem aus politischer Sicht voneinander getrennt sind: Ein Cluster bezieht sich auf die politisch rechtsorientierten alternativen Nachrichtenmedien des Typs I («Aufdecker der Mainstreamlügen»), die am weitesten von jenen alternativen Nachrichtenmedien des des Typs IV («Die seriöse Alternative) distanziert sind. Typ II hingegen («Verschwörung und Spiritualität») schafft eine Verbindung zwischen den beiden grösseren Clustern, was darauf hindeutet, dass Themen mit verschwörungstheoretischem Bezug nicht unbedingt politisch gefärbt sein müssen und in sehr unterschiedlichen Communities Anklang finden. Auch Typ III («Aufstand der Zivilgesellschaft) nimmt fallweise eine verbindende Rolle ein. Ein ähnliches Bild ergibt sich auch bei der gesamthaften Betrachtung des Retweet-Netzwerks alternativer und professioneller Nachrichtenmedien. Auch hier sind die Typen alternativer Nachrichtenmedien in ähnlicher Weise voneinander distanziert, obgleich das Netzwerk in sich geschlossener auftritt. Dies ist in erster Linie deswegen der Fall, weil «Mainstreammedien» zwischen den beiden polarisierten Communities alternativer Nachrichtenmedien eine verbindende Rolle einnehmen. Dabei untergliedert sich der «Mainstream», also die professionellen Nachrichtenmedien, basierend auf deren Länderzugehörigkeit in Communities. Insgesamt lässt sich die in Kapitel VII.3 aufgestellte These 1, wonach sich typenabhängige Communities alternativer Nachrichtenmedien bilden, bestätigen. Dies trifft vor allem für die - am stärksten voneinander 
kontrastierten - Typen I und IV zu, während Typ III rein quantitativ zu selten in Erscheinung tritt, um eine eigene Community abzubilden. Typ II ist vor allem im Netzwerk alternativer Nachrichtenmedien als eigene Community ausgeprägt, während er im Gesamtnetzwerk nicht eindeutig zugeordnet werden kann.

Wie auch bei den Follower-Netzwerken ist - rein quantitativ betrachtet unter den Communities alternativer Nachrichtenmedien Typ I («Aufdecker der Mainstreamlügen») am stärksten ausgeprägt (These 2). Bei diesem Befund muss das Sampling der Nachrichtenmedien berücksichtigt werden, bei dem der Typ I allenfalls aufgrund der Suchstrategie am prominentesten erschien (vgl. Limitationen; Kapitel VIII.2). Dennoch ist - auch aufgrund des aktuellen Forschungsstandes, der sich fast ausschliesslich auf alternative Nachrichtenmedien dieses Typs richtet, - davon auszugehen, dass neben professionellen Nachrichtenmedien vor allem alternative Nachrichtenmedien mit einer rechtspopulistischen Grundhaltung und dem selbst auferlegten Ziel, «Lügen des Mainstreams» aufzudecken, resonanzreich sind und (zumindest) in der deutschen Twittersphäre an Deutungsmacht gewinnen. Dies kann auch an den hohen Graden zentraler Knoten abgelesen werden (z. B. Tichys Einblick), die teilweise mit jenen professioneller Nachrichtenmedien vergleichbar sind.

These 3, die von einer Vernetzung der unterschiedlichen Alternativmedientypen mit politischen Akteur_innen gleicher Gesinnung ausgeht, kann auch anhand der Retweet-Netzwerke bestätigt werden. Insbesondere bei Typ I liess sich erneut feststellen, dass dieser Typ mit rechtspopulistischen Akteur_innen aus allen drei Untersuchungsländern in enger Relation steht. $\mathrm{Zu}$ nennen sind hier in erster Linie die AfD und ihre politischen Repräsentant_innen wie auch AfD-Unterstützer_innen oder aber beispielsweise der Schweizer Rechtspopulist Roger Köppel mit seinem Nachrichtenmedium Die Weltwoche. Österreichische Politiker_innen aus dem rechten politischen Spektrum stellen keine zentralen Knotenpunkte in der Twittersphäre des DACH-Raums dar und scheinen über andere Kanäle ihre Zielgruppen anzusprechen. Politisch linksorientierte Politiker_innen der drei Länder sind tendenziell eher in den Communities des medialen «Mainstreams» vorzufinden oder allenfalls in der Nähe alternativer Nachrichtenmedien der Typen III und IV.

These 4 postuliert eine unterschiedliche Vernetzung bzw. Distanz zwischen alternativen Nachrichtenmedien und dem medialen und politischen Establishment. Diese These kann am besten unter Bezugnahme auf das Gesamt-Retweet-Netzwerk alternativer und professioneller Nachrichtenmedien interpretiert werden. Dabei zeigt sich vor allem, dass die alternativen Nachrichtenmedien der Typen III und IV Teile der Ländercommunities des medialen und politischen «Mainstreams» sind, während Typ I in sich geschlossen am wenigsten mit dem «Mainstream» vernetzt zu sein scheint. Inwiefern dennoch Relationen zwischen diesem alternativen Nachrichtenmedientyp und medialen und politischen Eliten 
existieren, wird im interpretativen Teil der Netzwerkanalyse (Kapitel VII.4) erörtert.

\section{VII.3.3 Zwischenfazit: Netzwerkstrukturen alternativer Nachrichtenmedien}

Die Begrifflichkeit «alternative Nachrichtenmedien» impliziert selbst ein relationales Verhältnis, zumal eine «Alternative» nur in Bezug zu einer Opposition eingenommen werden kann. Insofern eignen sich netzwerkanalytische Verfahren, um diesen Relationen auf den Grund zu gehen. Die Analyse der deutschsprachigen Twittersphäre bietet aufgrund ihrer vor allem journalistischen und politischen Ausrichtung (Rauchfleisch \& Metag, 2016) eine sehr gute Datenbasis. Followerstrukturen geben Aufschluss über das Publikum und deren Newsrepertoires, zudem zeichnen sich diese durch eine hohe Beständigkeit über längere Zeit hinweg aus (Rauchfleisch et al., 2020). Retweetstrukturen zeigen hingegen, welche Akteur_innen über Retweets, also geteilte Beiträge untereinander, miteinander in Verbindung stehen. Die insgesamt vier Netzwerke belegen deutlich, dass es zwischen den resultierenden Follower- und Retweetcommunities starke Überschneidungen gibt. Auffallend bei den Netzwerken der alternativen Nachrichtenmedien (ohne Einschluss professioneller Nachrichtenmedien) ist jedoch, dass die Retweetstrukturen im Vergleich zu den Followerstrukturen wesentlich disperser sind. Während die Publika alternativer Nachrichtenmedien stärker miteinander verbunden sind, findet der Diskurs im Sinne von Retweets eher in getrennten Kammern statt. Als ähnlicher erwiesen sich hingegen die Follower- und Retweetcommunities, die aus dem Gesamtdatensatz (alternative und professionelle Nachrichtenmedien) resultierten. Während sich die Publika und Retweets alternativer Nachrichtenmedien der Typen II, III und IV stärker in die Communities des «Mainstreams» eingliedern, sind es vor allem die alternativen Nachrichtenmedien des Typs I - die «Aufdecker der Mainstreamlügen»-, die eigene Communities abbilden. Besonders relevant ist dabei der Befund, dass, bezogen auf die gesamten Netzwerke, alternative Nachrichtenmedien dieses Typs (vor allem aus Deutschland) einen relativ hohen Stellenwert einnehmen. Dies bedeutet, dass selbst unter Berücksichtigung des medialen «Mainstreams», also professionellen Leitmedien, alternative Nachrichtenmedien eine beachtliche Rolle in der Twittersphäre einnehmen. Sie können entsprechend - aus normativer wie auch analytischer Sicht - nicht als gesellschaftliches Randphänomen betrachtet werden, dem keine Aufmerksamkeit geschenkt werden muss. Vielmehr wird der Eindruck erweckt, dass die «Alternative» zunehmend den «Mainstream» erreicht - im Sinne von Deutungsmacht und Reichweitenzahlen ${ }^{148}$.

148 Reichweitenzahlen im Sinne von Twitter-Abonnements, aber auch anderen Social-MediaAuftritten wie auch monatlichen Seitenaufrufen (vgl. Kapitel VII.1). 
Dieses Kapitel leistete einen deskriptiven Überblick über die Netzwerkstrukturen und vervollständigte damit einen wesentlichen (Zwischen-)Schritt der vorliegenden Arbeit. Erstens konnte dadurch die typologische Einordnung alternativer Nachrichtenmedien bestätigt werden, die sich in Form von Communities weitgehend widerspiegelte. Zudem wurde offensichtlich, dass Aufklärungs- bzw. Media-Watchdog-Plattformen, die sich vor allem mit dem medialen Metadiskurs oder der Faktenprüfung beschäftigen (z. B. Übermedien, Correctiv.org etc.), weitere deutungsmächtige Akteur_innen in der Twittersphäre darstellen. Sie fallen zwar nicht eindeutig unter die in dieser Studie verfolgten Kriterien alternativer Nachrichtenmedien, die vorab so definiert wurden, dass regelmässig (in mindestens zweiwöchentlichen Abständen) Nachrichten publiziert werden, dennoch agieren sie als eine weitere Form von «Alternativmedien» oder besser alternativer Plattformen, indem sie sich fernab des medialen und politischen Mainstreams das Ziel setzen, z. B. potentielle Falschnachrichten aufzudecken oder zu überprüfen und das - im Gegensatz zu Typ I - basierend auf journalistischen Qualitätskriterien, ohne dabei selbstständig Nachrichten zu verbreiten. Was die typologische Einordnung angeht, wären diese alternativen Plattformen basierend auf ihrem Grundverständnis am ehesten in Typ IV («Die seriöse Alternative») einzuordnen. Zweitens konnten durch die netzwerkanalytische Betrachtung deutungsmächtige Akteur_innen in der Twittersphäre identifiziert werden - darunter vor allem interessant starke Knotenpunkte in Form alternativer Nachrichtenmedien, die in weiteren Analysen genauer untersucht werden können. Es stellen sich nun Anschlussfragen wie beispielsweise nach den Gründen für die jeweilige Deutungsmacht und nach den Kommunikationslogiken dieser Akteur_innen. Drittens geben Netzwerke auch inhaltlichen Aufschluss über Verbindungen bzw. Beziehungen oder Relationen zwischen Akteur_innen. Gleichzeitig impliziert eine deskriptive Analyse von Netzwerkstrukturen eine nur beschränkte Aussagekraft. Obwohl durch netzwerkanalytische quantitative Verfahren Communities identifiziert und Verbindungen zwischen unterschiedlichen Communities und einzelnen Akteur_innen aufgezeigt werden können, reicht eine reine Beschreibung dieser Strukturen nicht aus, um die Bedeutung hinter diesen Relationen herauszudestillieren. Relationen beruhen auf Narrativen zwischen Akteur_innen, genauso wie Communities nicht sinnleere Kreise repräsentieren. Das Retweeten oder Liken von Beiträgen muss gleichzeitig nicht bedeuten, dass die publizierten Inhalte der Absender_innen befürwortet werden. Die quantitative Netzwerkanalyse bietet zwar einen guten Überblick über Strukturen und Relationen, das Warum bleibt allerdings aussen vor. Aus diesem Grund erfolgt die interpretative Auswertung der Akteursrelationen im sich anschliessenden Kapitel. Die zentrale Frage richtet sich demnach nicht auf die Netzwerkstrukturen, sondern die inhaltlichen Bezugnahmen zwischen Akteur_innen. 